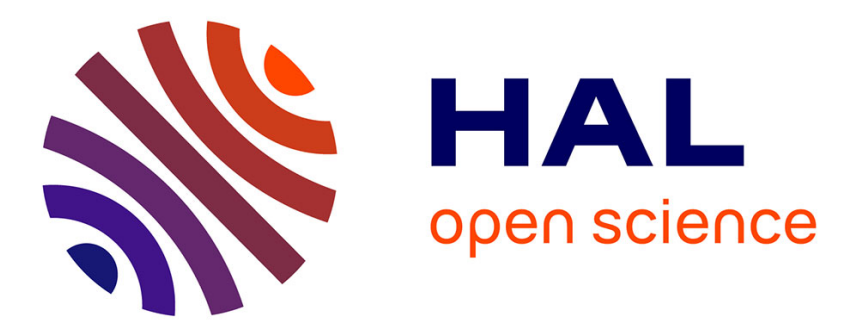

\title{
Aux origines de la LGV Rhin-Rhône : le rôle de la Suisse dans les logiques argumentatives des stratégies ferroviaires dans le Nord-Franche-Comté (XIXe-XXIes.)
}

Robert Belot, Pierre Lamard

\section{- To cite this version:}

Robert Belot, Pierre Lamard. Aux origines de la LGV Rhin-Rhône : le rôle de la Suisse dans les logiques argumentatives des stratégies ferroviaires dans le Nord-Franche-Comté (XIXe-XXIes.). RTS

- Recherche Transports Sécurité, 2014, Grande vitesse ferroviaire et territoires, 2013 (03), pp.211-227. 10.4074/S0761898013003051 . hal-01670631

\section{HAL Id: hal-01670631 \\ https://hal.science/hal-01670631}

Submitted on 21 Dec 2017

HAL is a multi-disciplinary open access archive for the deposit and dissemination of scientific research documents, whether they are published or not. The documents may come from teaching and research institutions in France or abroad, or from public or private research centers.
L'archive ouverte pluridisciplinaire HAL, est destinée au dépôt et à la diffusion de documents scientifiques de niveau recherche, publiés ou non, émanant des établissements d'enseignement et de recherche français ou étrangers, des laboratoires publics ou privés. 


\title{
Aux origines de la LGV Rhin-Rhône : le rôle de la Suisse dans les logiques argumentatives des stratégies ferroviaires dans le Nord-Franche-Comté $\left(\mathrm{XIX}^{\mathrm{e}}-\mathrm{XXI}^{\mathrm{e}} \mathrm{s.}\right.$
}

\author{
The origins of the Rhine-Rhône high-speed line: \\ Switzerland's role in the logic of rail strategy in Nord-Franche-Comté (19th to 21st Century)
}

\author{
Robert Belot · Pierre Lamard
}

Reçu le 28 septembre 2012 ; accepté le 28 octobre 2013

(C) IFSTTAR et Éditions NecPlus 2013

\begin{abstract}
Résumé Une approche historienne révèle que la question du TGV Rhin-Rhône s'inscrit dans une relation partenariale séculaire avec la Suisse, plus particulièrement avec sa partie jurassienne et francophone. Dès le milieu du XIX ${ }^{\mathrm{e}}$ siècle, le paradigme helvétique s'insère au cœur des politiques de développement du ferroviaire dans cette marche de l'Est que constitue le Nord Franche-Comté. Une communauté de destin et d'intérêt entre des espaces de marge se consolide au gré des dossiers d'infrastructures majeures. L'épaulement franco-suisse a joué un rôle majeur dans le processus d'appropriation politique de la LGV. S'il répond à une volonté de mise en résonance de ces territoires à la culture transfrontalière ancienne, il correspond à la nécessité d'une inscription dans la dynamique des grandes transversales européennes (Nord-sud et Est-Ouest). Cet emboîtement des intérêts régionaux et transnationaux constitue la trame de ce projet, même si, initialement, semble dominer une tendance obsidionale autour de la lutte contre «l'enclavement » et « l'encerclement ». Jean-Pierre Chevènement, l'inspirateur et l'acteur multi-scalaire de la LGV, a très bien su jouer sur ce sentiment largement partagé. C'est pourquoi, dès l'origine, il a associé la Suisse et encouragé son désir d'appariement au réseau français de la grande vitesse, une manière aussi pour lui de dépasser le tropisme allemand.
\end{abstract}

Mots clés territoire - ferroviaire - politique · transfrontiérité franco-suisse $\cdot$ enclavement

\footnotetext{
Robert Belot $(\bowtie)$

Université de Belfort-Montbéliard

Laboratoire IRTES-RÉCITS (EA 7274)

90010 Belfort Cedex

robert.belot@utbm.fr

Pierre Lamard $(\triangle)$

Université de Belfort-Montbéliard

Laboratoire IRTES-RÉCITS (EA 7274)

90010 Belfort Cedex

pierre.lamard@utbm.fr
}

\begin{abstract}
A historical approach makes it clear that the question of the Rhine-Rhône high-speed line is bound up with an age-old partnership with Switzerland, and particularly its French-speaking and Jura areas. Since the mid-nineteenth century, the Swiss paradigm has been at the heart of rail development policy in the eastern marches of northern Franche-Comté. The shared destinies and interests of the border areas were consolidated through major infrastructure projects. French-Swiss support has played an important role in the process of political appropriation of the high-speed line. While reflecting a desire to bring these territories closer together, with their ancient cross-border culture, it also corresponds to the need for representation in the dynamic of the major trans-European routes (northsouth and east-west). This intermingling of regional and trans-national interests constitutes the guiding thread of the project, even if a siege mentality concerned with countering "isolation" and "encirclement" initially seemed to dominate. Jean-Pierre Chevènement, the inspiration and multi-level advocate for the high-speed line, has successfully taken advantage of this widespread feeling. This is why he involved Switzerland from the beginning and encouraged its desire to connect with the French high-speed network. For him it is also a way of overcoming the German bias.
\end{abstract}

Keywords Territory $\cdot$ Railways $\cdot$ Politics $\cdot$ Franco-Swiss relations · Isolation

\section{Introduction}

Inaugurée en décembre 2013, la LGV (Ligne à Grande Vitesse) Rhin-Rhône est une expérience très intéressante pour nourrir la réflexion sur la question de la concurrence/ congruence des territoires et de la place des villes moyennes dans la genèse de l'accès des territoires à la grande 
vitesse. Elle est aussi un cas exemplaire qui permet de mieux renseigner les logiques d'acteurs et de réseaux, la complexité actorielle, les phénomènes de représentations et d'appropriation et le processus de décision politique dans les grandes infrastructures ferroviaires.

On aime à dire que la LGV Rhin-Rhône a une mère, la SNCF (Société nationale des chemins de fer français), et beaucoup de pères Parmi ses pères, l'un des premiers d'entre deux est sans conteste Jean-Pierre Chevènement, élu de Belfort et de son Territoire et maintes fois ministre. Il a été, pourrait-on dire, la locomotive et l'acteur multiscalaire de ce projet majeur, dont la gestation a duré 30 ans. Il convient à présent de repenser ce projet dans le cadre de la dynamique territoriale de l'Aire urbaine Belfort-Montbéliard où il a pris naissance et, ce faisant, de réinterroger la problématique de « l'effet structurant» des infrastructures sur le territoire et l'économie. Le fonds privé Jean-Pierre Chevènement (auquel nous avons été les premiers à accéder [1] $]^{1}$ ) apporte des éléments nouveaux et importants sur l'origine du TGV (Train à Grande Vitesse) Rhin-Rhône, à travers l'association internationale «Trans-Europe TGV » qu'il a fondée en mai 1986 et dont le siège était à Belfort.

Ces archives nous permettent de dévoiler ce que furent les grandes lignes argumentatives du projet dans sa phase primitive mais aussi les ambitions géopolitiques des acteurs et le rôle que la Suisse a joué dans le processus de crédibilisation-légitimation de cette aventure. L'utilisation de la Suisse n'a pas été seulement un effet d'opportunité. La prise en compte du partenariat de la Confédération helvétique s'inscrit dans un temps beaucoup plus long et se retrouve, dès le milieu du XIX ${ }^{\mathrm{e}}$ siècle, au cœur des politiques françaises de développement du ferroviaire dans cette marche de l'Est que constitue le Nord-Franche-Comté.

Un regard rétrospectif permet d'éviter une trop grande polarisation sur un acteur, aussi déterminant qu'ait pu être son rôle, et d'accéder à la profondeur des processus qui participent au devenir et aux représentations des territoires et qui révèlent des postures séculaires lisibles géographiquement. Il existe des rationalités peu perceptibles échappant à un cadre d'analyse traditionnelle mais qui éclairent des stratégies plus infra et des plus durables. C'est pourquoi la construction argumentative de la LGV Rhin-Rhône relève à la fois de logiques micro-régionales et de tropismes qui excèdent le schéma hexagonal. En effet, l'existence d'un premier barreau grande vitesse qui

\footnotetext{
${ }^{1}$ Il s'agit du fonds personnel de l'ancien ministre, déposé aux Archives municipales de Belfort (AMB). Il contient des notes de travail, des lettres, des documents publiés, des articles de presse. Les dates extrêmes sont 1986 et 2001. Les documents non référencés cités dans le présent article proviennent de ce fonds. D'autres fonds, déposés aux Archives départementales du Territoire de Belfort (ADTB) ou aux archives municipales, ont été dépouillés. À noter que le fonds de l'association Métropole Rhin-Rhône est abrité par les ADTB.
}

induit des liaisons transeuropéennes sans passer par Paris, implique une focale d'analyse qui passe par la prise en compte d'une dimension internationale avec pour ambition l'ouverture de l'Europe rhénane sur l'Europe du Sud. Mais le projet de la grande vitesse ferroviaire est rythmé par une autre chronique, plus subtile, celle de la petite vitesse et de ses synergies locales qu'un regard sur le temps long permet de décrypter.

Rares sont les réflexions dédiées à la grande vitesse ou aux grandes infrastructures ferroviaires transeuropéennes qui prennent appui sur une démarche historienne. Pourtant, il a déjà été montré de manière magistrale l'intérêt heuristique que revêt une approche historienne pour, par exemple, contrebattre le mythe de la causalité linéaire de l'effet ferroviaire [2]. Ici, le recours à l'histoire se justifie d'autant plus que la stratégie des acteurs en tire argument pour mettre en avant une source de légitimité qui permet d'échapper à l'habituelle rhétorique économique du discours politique. L'essence d'un tel projet ne peut se révéler sans puiser dans une temporalité plus lente qui a le mérite de faire apparaître des lignes de force qui dépassent la jauge temporelle de la décennie et qui influent sur des logiques et des imaginaires d'alliances et de stratégies [3].

Le détour par la Suisse et le recours à l'histoire permet de dévoiler une constante séculaire qui a été réactivée dans la phase initiale du projet LGV : la quête d'une transfrontiérité ferroviaire efficiente pour dompter l'Arc Jurassien.

\section{D'une posture obsidionale au mythe des diagonales européennes}

\section{Une infrastructure pensée comme l'élément d'une stratégie d'appropriation territoriale}

La rhétorique argumentative de Jean-Pierre Chevènement pourrait passer comme une illustration de l'engouement saint-simonien des hommes politiques français qui les conduit souvent à user et abuser du mythe de l'effet structurant des infrastructures de transport sur les modes d'organisation territoriale et sociétale. Il serait réducteur de regarder son discours comme un simple effet de la doxa déterministe de la «causalité linéaire » qui ne se soucie pas de prendre en compte la validité de ses énoncés, bien que les recherches sur la question de l'impact socio-économique du TGV se déploient dans le milieu des années 1980. La pensée résolument techniciste de Chevènement s'apparie à une approche politique qui pense en termes d'histoire, de globalité et d'interaction. Elle prend en compte la spécificité d'un territoire marqué depuis 1879 par l'industrie des transports et de l'énergie. Sa culture historique permet à l'élu de Belfort de comprendre que les infrastructures 
de transport ont « suivi le cycle de l'activité industrielle plus que l'inverse $»^{2}$. Sa décision de créer une université de Technologie, de manière concomitante au lancement du projet $\mathrm{TGV}$, témoigne de cette mise à distance du « mythe de l'effet »[6,7]. Sa volonté d'installer la gare TGV à mi-parcours de Belfort et de Montbéliard, comme l'UTBM (Université de Technologie de Belfort-Montbéliard) ou l'hôpital médian, et de construire une zone d'activités autour de la nouvelle gare (justement appelée la «JonXion») (Maquette 1) procède d'une démarche stratégique qui entend anticiper et accompagner de manière dynamique l'interaction entre le transport et le territoire ${ }^{3}$. Le « $\mathrm{X}$ » étrange qui barre le nom de cette gare représente les quatre points cardinaux et symbolise la volonté des politiques locaux de faire de la LGV un instrument de politique territoriale et de rayonnement intra, inter et extra-régional. En effet, contrairement à la gare de Besançon (la capitale franc-comtoise n'ayant pas vocation à constituer un pivot $\mathrm{du}$ développement régional ${ }^{4}$ ), celle de l'Aire urbaine Belfort-Montbéliard " dessert des territoires situés aux quatre points cardinaux par le chemin de fer » grâce aux lignes Belfort-Delle et Épinal-Belfort. Le projet de réhabilitation de l'axe ferroviaire Nancy-Épinal-Belfort soutenu par les politiques ${ }^{5}$ vise justement à connecter la Lorraine et la Haute-Saône (qui souffre à présent d'enclavement [9]) à la LGV Rhin-Rhône et celle-ci au TGV Est-européen, ce qui devrait avoir comme effet de transformer la Franche-Comté de corridor en carrefour.

Dès l'origine, le projet de grande vitesse ferroviaire a été pensé dans une logique d'appropriation régionale. C'est d'ailleurs pourquoi le « grand canal » n'a pas été préféré à la LGV. L'ambition transeuropéenne aurait pu trouver une réponse dans le projet du Canal Rhin-Rhône, déclaré d'utilité publique en 1978. C'était l'occasion de relier Rotterdam et Fos-sur-Mer, l'Europe rhénane et l'Europe

\footnotetext{
${ }^{2}$ Il a été démontré depuis que le retour sur investissement local de la création d'une infrastructure de transport, loin d'être automatique, ne peut avoir lieu sans la mise en œuvre d'une politique de développement. Voir[4, 5].

${ }^{3}$ Le conseil général du Territoire de Belfort et la Communauté d'agglomération belfortaine $(\mathrm{CAB})$ ont financé la création d'une ZAC (zone d'aménagement concerté) TGV. La CAB a également engagé la création d'une ZAC sur l'ancien Dépôt-atelier de munitions spéciales (DAMS) sis sur le territoire des communes de Meroux et de Bourgogne, en contact avec la ZAC «TGV » : ce sera le Parc d'innovation des Plutons. D'une superficie de 30 hectares, ce Parc sera relié à l'autoroute $\mathrm{A} 36$ et bordé au Nord par la voie ferrée Belfort-Delle-Delémont. Il s'agit de favoriser l'implantation d'activités et de services économiques relevant du secteur tertiaire supérieur. À noter que la $\mathrm{CAB}$ a participé au financement du premier tronçon de la branche Est de la LGV Rhin-Rhône entre Auxonne et Petit-Croix $(9,21$ millions d'euros).

${ }^{4}$ Contrairement, par exemple, à Lille. Voir [8].

${ }^{5}$ Il s'agit essentiellement de l'électrification de la ligne EpinalBelfort.
}

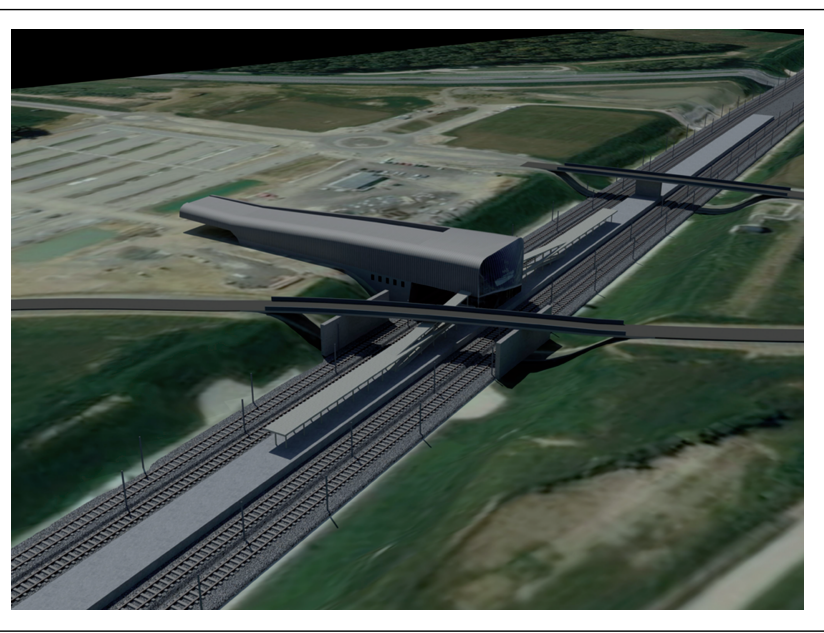

Maquette 1 Maquette 3D de la gare TGV de Belfort-Montbéliard (2012) réalisée par la société Voxelia avec le soutien du laboratoire IRTES-SET, travaux issus du projet de simulateur ferroviaire FLO en collaboration avec Alstom

méditerranéenne. On sait que la victoire de François Mitterrand en 1981 met en sommeil ce projet, fortement critiqué par les écologistes, dont la rentabilité financière n'a pas été prouvée [10]. À l'échelon local, les décideurs politiques rendent un arbitrage précoce en faveur du TGV. Un homme comme Christian Proust, très lié à Jean-Pierre Chevènement, président du Conseil général du Territoire de Belfort de 1982 à 2004, membre du Conseil régional de Franche-Comté pendant 18 ans, ne croit pas en la capacité du " grand canal » à constituer un facteur déterminant d'aménagement du territoire. Il aime à citer la canalisation de la Moselle, dont il est originaire, pour exprimer son scepticisme sur la question des maillons de grandes infrastructures. Il est persuadé que cette liaison fluviale ne pouvait produire que des " développements en haltère » impuissants à nourrir l'ensemble de l'axe ${ }^{6}$. Pour Jean-Pierre Chevènement et les plus de gauche, il n'était pas envisageable de financer deux projets d'infrastructures d'envergure.

\section{Peur franc-comtoise de «l'enclavement » et crainte suisse du « contournement »}

Alors que l'on pourrait penser que l'inspirateur de ce projet soit prisonnier d'un tropisme local ou national, ce qui frappe dès l'abord est la dimension internationale de la démarche. Du moins est-ce ainsi présenté dans les premiers

\footnotetext{
${ }^{6}$ Témoignage de Christian Proust à la table ronde organisée dans le cadre du colloque « Les transports au cœur des territoires et de la société ». Voir [3, p. 105]
} 


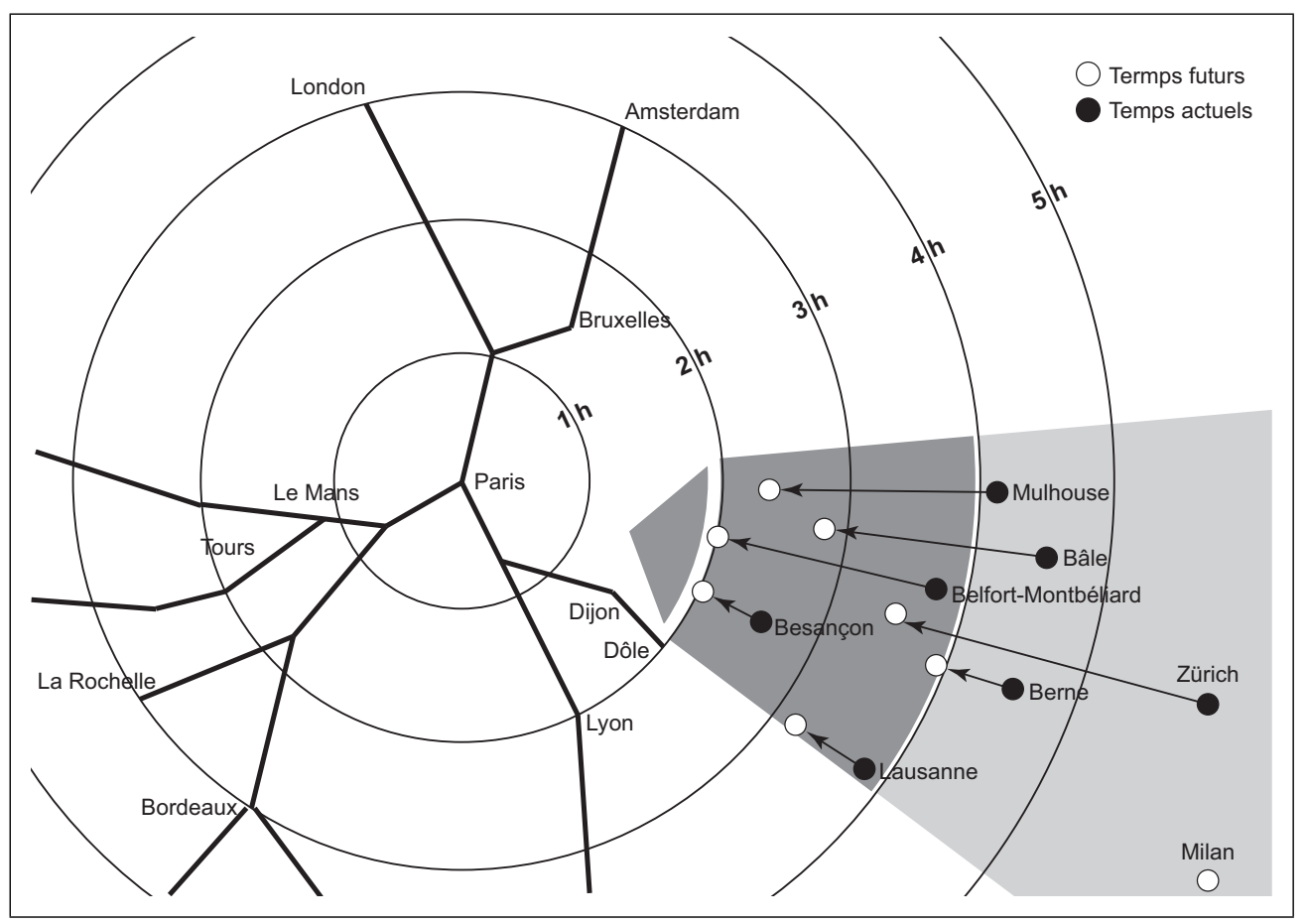

Fig. 1 Schéma des couronnes concentriques autour du «pôle d'influence » de Paris visant à montrer que la Franche-Comté, le Sud de l'Alsace, la Suisse septentrionale (Bâle) sont extérieurs à la $3^{\mathrm{e}}$ couronne (distance-temps supérieur à 3 heures de Paris) et que Zürich est exclue de ce pôle d'influence

Ce schéma apparaît dans le premier document « Le TGV France Suisse » (1998) ? Reproduction J.-P. Droux, CRESAT, UHA.

documents conçus par l'association « Trans-Europe TGV ». L'ambition ultime est la construction d'un réseau grande vitesse centre-Europe et l'avènement d'un « vaste réseau européen interconnecté, à l'instar du réseau autoroutier ». L'association internationale « Trans-Europe TGV » évoque l'Allemagne et l'Italie, mais surtout la relation francosuisse, s'appuyant sur le plan Rail 2000 qui vise alors l'ouverture de $120 \mathrm{~km}$ de lignes selon une conception en réseau avec les lignes existantes.

Le plus vieux document du fonds Chevènement (1988) s'intitule : « Le TGV France-Suisse ». Il est bilingue : français et allemand. Ce document met en valeur le fait que le réseau européen des infrastructures à grande vitesse en train de naître ignore « complètement » la Franche-Comté, le sud de l'Alsace et la Suisse (dans l'esprit des rédacteurs, il s'agit de Bâle et de Zürich). Un territoire qui est présenté comme déficitaire sur le plan ferroviaire mais aussi en termes identitaires. Jean-Pierre Chevènement veut montrer au contraire que cet espace présente «un tissu de complémentarités industrielles et culturelles très prometteuses » et représente un poids financier (Zürich), culturel, universitaire et touristique (Suisse).

D'emblée, dans l'esprit de son premier promoteur, la ligne à grande vitesse est adossée à l'ambition de mieux conjoindre trois territoires (Franche-Comté, Alsace et
Suisse), de les engager sur le chemin de la transfrontiérité ${ }^{7}$ et de l'interterritorialité et de les rapprocher de Paris.

D'où la volonté, qui transparaît dans les documents d'époque, de faire apparaître la réalité et l'intérêt d'une communauté de destin entre ces trois espaces non desservis par le $\mathrm{TGV}^{8}$ (Fig. 1) qui sont présentés comme courant un risque d' $d$ enclavement ». La congruence est préférée

\footnotetext{
${ }^{7}$ Sur la question de la transfrontiérité franco-suisse, le laboratoire RÉCITS/UTBM a déjà initié des recherches. Voir [11, 12, 13, 14].

${ }^{8}$ À l'exception de la liaison TGV mise en place entre Paris et Lausanne le 22 janvier 1984 exploitée conjointement par la SNCF et les chemins de fer fédéraux suisses. Le document " Le TGV France-Suisse » l'ignore, attaché à mettre en valeur le problème de la troisième couronne qui correspond à une distance-temps supérieure à 3 heures de Paris, et le repère qui menace la quatrième couronne (Zürich) vouée à devenir « espace résiduel ». Cette absence n'est pas neutre. Dans sa lettre au Premier ministre du 13 février 1989 ( $c f$. plus loin), Jean-Pierre Chevènement cible les sous-ensembles de ces régions qui intéressent son projet : il s'agit du Sud de l'Alsace, du Nord-Franche-Comté et du triangle Zürich-Baden-Bâle. Mais c'est surtout Belfort-Montbéliard qui requiert en fait toute son attention, comme il le laisse apparaître clairement dans cette lettre : « Comment expliquer que des villes comme Grenoble, Chambéry, ou bien Marseille, Toulon ou bien Lausanne, Neuchâtel et Berne, de l'autre côté du Jura, soient desservies par le TGV, alors que Belfort et Montbéliard, pourtant plus proches des lignes à grande vitesse existantes, ne le sont pas? »
} 
à la concurrence. Mais en fait, la posture semble relever initialement d'une attitude plus défensive que constructive où la problématique de l'émergence d'un territoire entre Rhône et Rhin apparaît marginalement. Pour la Suisse, explique-t-on, le danger est d'être « contourné » ${ }^{9}$ au Nord par un axe Paris-Strasbourg et, au sud, par un axe Modane-Turin. Bâle pourrait ainsi perdre sa position d'étoile ferroviaire d'importance européenne. Tout se passe comme si l'association « Trans-Europe TGV » prenait à son compte les intérêts de la Suisse !

L'association s'appuie sur le rapport de l'ingénieur général des Ponts-et-Chaussées Rattier (1985), chargé par le ministère français des Transports de réfléchir à un projet de liaison ferroviaire rapide entre Paris et l'Allemagne via la Lorraine, appelé TGV Est. En effet, les autorités françaises semblent plutôt intéressées par la relation franco-allemande, comme en témoigne, en 1987, le " Projet de liaison rapide Paris-Est de la France-Sud-Ouest de l'Allemagne ${ }^{10}$ [16]. Cette étude met en évidence le risque pour le Nord de la Franche-Comté de se situer à l'écart des liaisons ferroviaires rapides. Jean-Pierre Chevènement s'en émeut et attire l'attention du Gouvernement, lequel charge alors, dès janvier 1986, le même ingénieur Rattier d'une mission complémentaire sur la desserte par TGV de la Franche-Comté ${ }^{11}$.

Dans la foulée, le député-maire de Belfort décide de créer, en mai 1986, l'Association Trans-Europe TGV LondresParis-Aisy-Bâle-Adriatique, présidée par le député-maire de Mulhouse ${ }^{12}$. Peut-être est-ce son exemple qui conduit le maire de Lille, Pierre Mauroy, à créer lui aussi son association fin 1986. Mais cette association (TGV gare de Lille) a un but plus limité et répond d'abord à une manœuvre tactique visant à contourner la communauté urbaine hostile au projet [17]. Dans le cas du Lyon-Turin, l'entrepreneur politique (Louis Besson) s'appuie d'abord sur une institution, le conseil régional Rhône-Alpes [18]. Dans le cas qui nous occupe, l'association est un acteur majeur du portage du projet, mais aussi de son partage par les élus des autres collectivités (car ici trois régions sont concernées) et par la Suisse. C'est un outil de médiation,

\footnotetext{
${ }^{9}$ Sur la peur de la Suisse de subir un contournement ferroviaire et d'être écarté des courants de trafics européens. Voir [15].

${ }^{10} \mathrm{Ce}$ rapport évoque au contraire une baisse du trafic ferroviaire entre la France et la Suisse.

${ }^{11}$ Le second rapport Rattier sera remis en juillet 1986. Il reconnaît l'importance d'une « desserte des villes de la Franche-Comté du Nord », mais écarte la solution Aisy-Vesoul pour des raisons de rentabilité

${ }^{12}$ Le président de l'Association est Joseph Klifa, député-maire de Mulhouse, Jean-Pierre Chevènement étant vice-président.
}

mais aussi d'influence et de lobbying, qui a également l'avantage de montrer que Jean-Pierre Chevènement, dont le rôle est incontestablement moteur dans ce dossier, s'inscrit dans une démarche collective.

La raison d'être primitive de l'association est la réalisation d'une ligne TGV entre Aisy et Vesoul, permettant de relier le Nord-Franche-Comté, le sud de l'Alsace et la Suisse alémanique à la ligne TGV Sud-Est. L'Allemagne est évacuée du champs de réflexion stratégique, reflet d'une attention permanente que Jean-Pierre Chevènement a porté à l'Allemagne (surtout après 1989) et à ses tendances au « géocentrement » et à la «métanoïa » [19]. L'intégration de la problématique helvétique intervient à ce moment pour contrebalancer le tropisme allemand. La thèse de l'enclavement est reprise côté suisse dans le rapport Boillat ${ }^{13}$ (1987), du nom de ce chef du service Transport et Énergie du canton du Jura. Alain Boillat critique l'insuffisante prise en compte du potentiel de clientèle helvétique. On sait que les cultures ferroviaires françaises et suisses ne sont pas identiques. Il faudra du travail pour que la philosophie TGV (« aussi vite que possible ») s'accorde avec la philosophie suisse Rail 2000 (« aussi rapidement que nécessaire »). L'idée-force est de concevoir une approche «système » à travers la création d'un réseau transfrontalier.

L'hypothèse de départ est reformulée grâce aux propositions de la Suisse. On part de l'idée d'un prolongement de la ligne LGV Sud-Est de $167 \mathrm{~km}$ et de la mise en place de deux branches d'interconnexion (conception en « $\mathrm{Y} »$ semblable au TGV Atlantique) :

\section{- l'une sur Dijon-Besançon-Dôle-Neuchâtel-Berne- Lausanne ; \\ - l'autre sur Vesoul-Belfort-(Montbéliard)-Mulhouse- Bâle-Zürich.}

C'est sur la base du rapport Rattier et, surtout, du rapport Boillat, que le premier document public de l'Association (« Le TGV France-Suisse ») est construit. Au cours de cette année 1988, le conseil régional de FrancheComté, qui apporte son concours financier, demande alors à la SNCF d'étudier cette hypothèse. On voit se constituer une configuration institutionnelle triangulaire qui servira de cadre à l'aventure : une association influente, relayée initialement par une collectivité réactive (la région Franche-Comté) ; l'État et la SNCF ; la Suisse.

\footnotetext{
${ }^{13}$ Présenté lors de l'Assemblée générale du 30 avril 1987.
} 


\section{L'émergence du mythe des « deux diagonales » européennes}

En 1989, Jean-Pierre Chevènement, alors ministre de la Défense, est au cœur de l'État français. Il est bien placé pour agir sur le Gouvernement qui a inscrit à l'ordre du jour du conseil des ministres (31 janvier 1989) une communication sur le développement du réseau des liaisons ferroviaires à grande vitesse en France et en Europe. Dans une lettre au Premier ministre ${ }^{14}$, il se fait " apprenti géographe », en expliquant qu'il faut s'attacher à traiter enfin « l'angle mort » entre Strasbourg et Lyon, et notamment la « trouée de Belfort », qu'il présente comme « le seul seuil naturel à basse altitude $(357 \mathrm{~m})$ entre la Méditerranée et la frontière allemande à travers la barrière naturelle des Alpes, du Jura et des Vosges ». La prise en compte de la Suisse est toujours présentée, car elle s'inscrit dans une logique d'emboîtement d'intérêt européen, national et régional. En tant que président de l'Association Aire Urbaine 2000 (Belfort-Montbéliard-Héricourt), il met en valeur le risque d'« enclavement » de la région Franche-Comté, « la plus industrielle de France et la plus exportatrice $»^{15}$.

Dans sa lettre au Premier ministre, l'élu franc-comtois préfère adopter un point de vue qui est à la fois plus panoramique (l'échelle est l'Europe et intègre l'Espagne pour la première fois) et plus réducteur (les flux de passagers). En puisant dans la rhétorique planologique des « faisceaux », « arcs » et autres figures géométriques, très en vogue à ce moment à la Datar (Délégation interministérielle à l'aménagement du territoire et à l'attractivité régionale) où l'on rêve de rééquilibrer l'Europe au Sud-Ouest, il recourt à la thèse des deux « diagonales » [20]. D'abord, la diagonale Allemagne-Méditerranée qui concerne le flux Nord-Sud. Il met en valeur la nécessité et l'opportunité de drainer les trafics entre l'Allemagne, le Midi de la France et la péninsule ibérique ${ }^{16}$. Le signe de cette évolution se retrouve dans l'évolution du titre de l'association : l'association Trans-Europe TGV se transforme en association Trans-Europe TGV Rhin-Rhône ${ }^{17}$. Un an après

\footnotetext{
${ }^{14}$ Lettre de Jean-Pierre Chevènement, ministre de la Défense, au Premier ministre, 13 février 1989.

${ }^{15}$ En fait, il plaide essentiellement pour le Nord-Franche-Comté, en recourant à un argument économique et social classique : depuis 1979, ce bassin de 300000 habitants a perdu 30000 emplois.

${ }^{16}$ Le gouvernement espagnol décide fin 1988 de construire des lignes nouvelles à écartement européen entre Lisbonne, Séville, Madrid et Barcelone, et d'acquérir des rames TGV.

${ }^{17}$ Les 12-13 octobre 1989, un colloque est organisé par le LET (Laboratoire d'économie des transports) de l'Université Louis Lumière sur le thème : "Les couloirs Rhin-Rhône dans l'espace européen. Quel avenir ? Quelles relations ? Quelles priorités ? ». Bien sûr, la chute du mur de Berlin renouvelle la donne géopolitique et réactive les problématiques liées aux échanges et au transport.
}

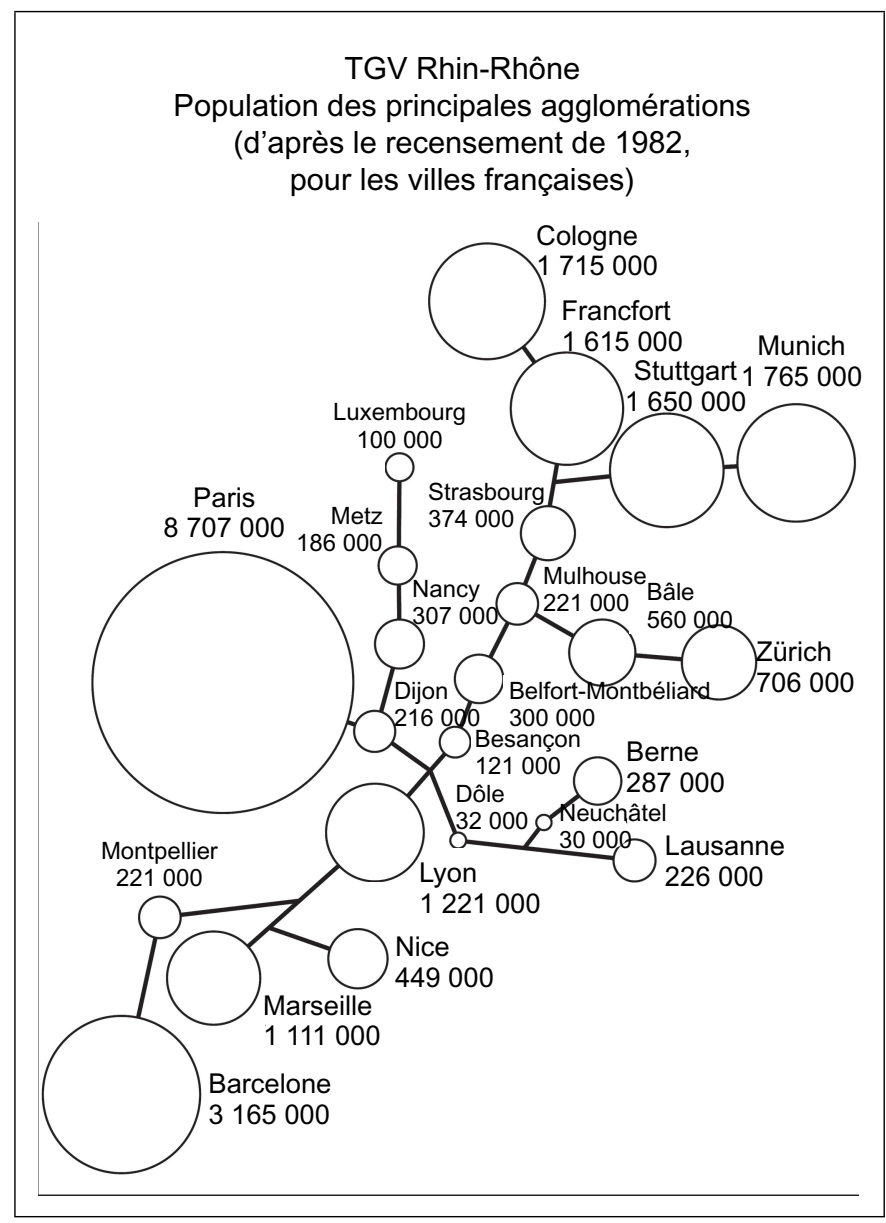

Fig. 2 Ce schéma spatialise les principales agglomérations qui seraient impactées par le TGV Rhin-Rhône. Il est tiré de la première étude (1990) qui vise à montrer que le TGV Rhin-Rhône constitue «l'un des maillons essentiels du réseau européen à grande vitesse »

Dans le schéma original, seule la ville de Belfort apparaît avec le chiffre de 75000 habitants, la ville de Montbéliard étant oubliée. Reproduction J.-P. Droux, CRESAT, UHA.

$\left(1990^{18}\right)$, dans une note intitulée « TGV Rhin-Rhône », Chevènement affirme, contre toute attente, que cet axe est porteur de développement « au fur et à mesure de l'intégration économique européenne dans le cadre du marché unique ${ }^{19}$. Au projet de TGV franco-suisse est associée la liaison TGV Rhin-Rhône-Méditerranée. Le projet assume désormais la nécessité d'un lien entre l'Europe rhénane et l'Europe méditerranéenne (Fig. 2).

La deuxième diagonale concerne l'Italie et l'Angleterre par la Suisse : le flux Est-Ouest. En fait, Chevènement craint

\footnotetext{
18 Jean-Pierre Chevènement devient président de l'association à cette date.

${ }^{19}$ Contre toute attente venant d'un homme qui a toujours été hostile à l'Acte unique.
} 
un tropisme rhénan et un écoulement du trafic vers l'Europe $\mathrm{du}$ Nord à cause, d'une part du projet de tunnel en Suisse sous les Alpes (Saint-Gothard) qui aboutira à Bâle ${ }^{20}$, d'autre part de la volonté des Allemands (en passe d'être réunifiés) d'étudier une liaison à grande vitesse de Bâle à Francfort, puis Cologne sur la rive droite du Rhin. Pour lui, la lutte contre l'enclavement Nord-franc-comtois passe la volonté de "désenclaver la Suisse alémanique, le triangle d'or Zürich-Baden-Bâle ». À le suivre, la Suisse craint les risques de « contournement » à l'échelle européenne; en effet, elle ne pourra réaliser chez elle une ligne TGV sur l'axe très peuplé Zürich-Berne-Genève (opposition des écologistes). Elle devrait voir avec intérêt la construction d'une ligne à grande vitesse à partir de Bâle vers Paris.

On voit que l'homme politique a affûté son argumentaire par une prise en compte de la problématique du rééquilibrage territorial français entre la ligne radiale, qui renforce le centralisme parisien, et l'axe méridien qui s'inscrit dans le temps long de l'histoire lotharingienne. Intuitivement et rapidement ${ }^{21}$, Chevènement a pris conscience de la tension qui caractérise le Grand-Est, qualifiée par Raymond Woessner de «région incomplète » [21].

L'association vise à obtenir la réalisation d'une ligne TGV en site propre de Mulhouse à Dijon, permettant les raccordements à la ligne du TGV Sud-Est. Mais c'est le double argument, de la vitesse et celui, largement invérifiable, alors de la «rentabilité » supposée d'une liaison ferroviaire à grande vitesse qu'il met in fine en exergue, "dès lors que seraient pris en compte à la fois le trafic de la Suisse vers Paris et celui en provenance de Francfort-Strasbourg vers le Midi de la France $»^{22}$ (Fig. 3).

\section{Le rôle de la Suisse dans la stratégie originelle visant à la création de la LGV Rhin-Rhône}

\section{Mise en place d'un lobbying institutionnel avec la Suisse}

Le message de Jean-Pierre Chevènement a fait son chemin au gouvernement. À l'automne de l'année 1990, le ministre des Transport, Michel Delebarre, consulte le Conseil fédéral suisse sur la présence de la Suisse dans le schéma directeur des TGV. La Suisse, par la voix de son ministre des

\footnotetext{
${ }^{20}$ Le projet d'élargissement à plusieurs voies du Saint-Gothard s'inscrit dans le cadre du projet des nouvelles ferroviaires à travers les Alpes. Il s'agit de créer une sorte d'autoroute ferroviaire à travers un tunnel ferroviaire de $57 \mathrm{~km}$. Le percement a été terminé en 2011.

${ }^{21}$ Car nous ne trouvons pas de références à des travaux théoriques dans le fonds Chevènement et de documents qui montreraient les raisons d'une évolution si rapide de son positionnement.

${ }^{22}$ Les premiers chiffres relatifs à son exploitation semblent confirmer son intuition.
}

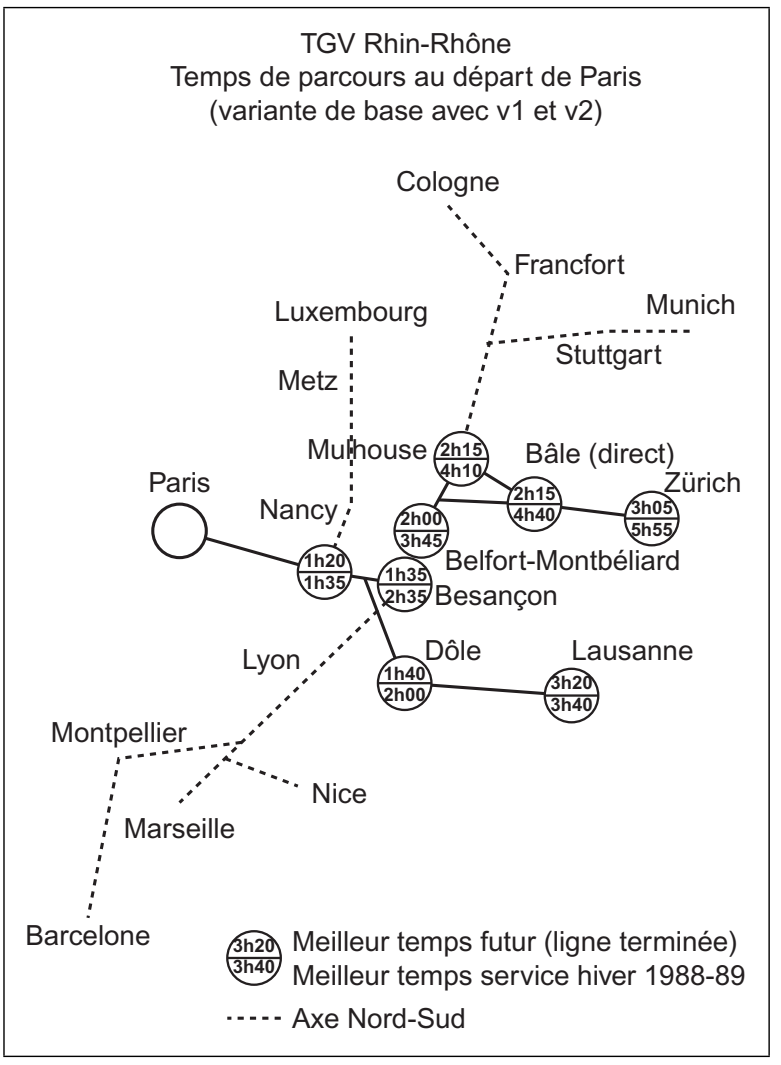

Fig. 3 Arbre à boules indiquant l'ordre de grandeur des gains de temps procurés par le projet Rhin-Rhône par rapport au meilleur temps du service d'hiver 1988-1989, tel qu'il apparaît dans la première étude de 1990

Reproduction J.-P. Droux, CRESAT, UHA.

Transports, prend position en faveur du TGV Rhin-Rhône. Deux mois après, le quatrième pôle de la configuration institutionnelle apparaît : en décembre 1990, le projet de TGV Rhin-Rhône est inscrit au schéma directeur européen ; il est présenté par les instances communautaires comme le maillon-clé du futur réseau européen de trains à grande vitesse. Le 14 mai 1991, le CIAT (Comité interministériel d'aménagement du territoire) inscrit le TGV Rhin-Rhône au schéma directeur français des liaisons ferroviaires. La bataille pour la reconnaissance vient d'être gagnée. Mais tout est à faire !

Le 14 juin 1991, au nom de l'association, Jean-Pierre Chevènement écrit au conseiller d'État du canton de Bâle, Mathias Feldges. Vantant les intérêts que représente le rattachement de la Suisse alémanique au réseau TGV, il demande que le canton de Bâle adhère à l'association Trans-Europe TGV et qu'il « sensibilise » les autres cantons alémaniques à cette démarche. Il s'agit de regrouper les volontés helvétiques pour donner du poids et de la légitimité internationale à l'action de l'association. Le but : créer une force de pression sur les acteurs français. Dans sa 
lettre au Conseiller d'État, le ministre le convie clairement à organiser une forme de lobbying institutionnel sur les gouvernements français et suisses : «Ensemble, cantons alémaniques et régions françaises, nous pourrons ainsi agir efficacement à deux niveaux. Sur le plan politique : intervention auprès des ministères des transports français et suisse pour l'ouverture le plus rapidement possible des discussions relatives à la réalisation du TGV Paris-Bâle ; sur le plan financier : contribution financière des régions concernées permettant d'apporter, dans des conditions à définir, une part de préfinancement à la première phase Dijon-Mulhouse à la condition que celle-ci puisse être opérationnelle avant l'an $2000 »$ [22]. Une pression permanente est nécessaire, explique Chevènement, car le schéma directeur arrêté par le gouvernement français n'a prévu « aucun ordre de priorité » et n'a pas retenu non plus l'idée d'une «certaine planification ». De la sorte, explique-t-il dans cette lettre, il est probable que la réalisation des projets TGV sera décidée " au coup par coup, en fonction des possibilités financières de l'économie française et de la SNCF ».

Le 31 octobre 1991, le président de l'association se rend à l'invitation de Madame Hedi Lang, Conseillère d'État (socialiste) du canton de Zürich chargée de l'économie publique. Un travail discret de «sensibilisation» dans les milieux zurichois avait été mené par le secrétaire général de l'Association. Un dossier, orienté du point de vue de la Suisse alémanique, a été constitué, traduit en allemand et diffusé dans les milieux politiques, économiques et dans la presse. Pour préparer sa visite, et lui montrer que les acteurs politiques et économiques zurichois sont favorables à son projet, la Chambre de commerce de Zürich publie une plaquette intitulée : Zürich Pro TGV. Wie weiter? [23]. L'occasion, c'est la célébration du $75^{\mathrm{e}}$ anniversaire de la fondation de la société Züblin AG. Cette société ${ }^{23}$ a eu l'idée d'éditer à ses frais une plaquette de promotion du $\mathrm{TGV}^{24}$. Jean-Pierre Chevènement participe à une conférence de presse présidée par Christian Boesch, directeur de la Chambre de Commerce de Zürich et à laquelle assiste Martin Imbach, président du conseil d'administration de la société Züblin, qui présenté sa plaquette. Nous n'avons pas retrouvé le discours du ministre, mais nous disposons d'une note faite à son attention comportant des « éléments de langage ». La ligne argumentative est la suivante : « Le projet de TGV Rhin-Rhône est un véritable TGV France-Suisse. Il permettra en effet de connecter la Suisse via Bâle-Zürich, via Lausanne, via Berne et Neuchâtel au

\footnotetext{
${ }^{23}$ Züblin AG se situe au $6^{\mathrm{e}}$ rang des sociétés suisses de travaux publics. Son principal actionnaire est la SIS Holding, groupe international travaillant dans le secteur de l'assurance-réassurance. Le groupe SIS France est installé à Paris.

${ }^{24} 6000$ exemplaires écoulés.
}

réseau TGV français et européen » [24]. Il convient de convaincre les Suisses que « le projet de TGV Rhin-Rhône constitue pour la Confédération helvétique un outil essentiel de rapprochement et de coopération avec la Communauté européenne ». Plus concrètement, pour Chevènement, l'objectif de cette journée est de « faire valoir la nécessité de créer une structure d'étude et de financement chargée d'apporter un préfinancement des études SNCF en ce qui concerne la première phase Mulhouse-Dijon/Dôle ». Il s'agit de proposer à la Suisse alémanique d'y participer, « en compagnie des régions françaises concernées ».

\section{La Suisse partante pour être raccordée au réseau TGV}

Les cantons romands et alémaniques se déclarent favorables. La présence de la Suisse apporte une légitimité supplémentaire au projet. L'argumentaire géopolitique en est renforcé. Mais il s'agit aussi d'une question financière. Le 18 décembre 1991, est ainsi créée une société d'économie mixte franco-suisse : la SEM Rhin-Rhône, société de financement et de planification. La SBS (Société de Banque Suisse) sera son conseiller financier, conjointement avec son partenaire côté français : la BNP. Les Suisses ne pouvaient qu'être acquis au principe de ce montage qui avait déjà servi dans le cadre du comité du Gothard. En font partie sept collectivités françaises et allemandes et six cantons suisses. Il s'agit de recueillir les fonds nécessaires aux études préalables et à la déclaration d'utilité publique, dans un premier temps, et de proposer un montage financier pour la réalisation de la première phase, dans un deuxième temps. Dans la foulée, la Communauté Européenne décide d'allouer 3,6 millions de francs suisses pour donner une impulsion aux études de réalisation.

Pour matérialiser son engagement, la SBS fait paraître un numéro spécial de sa revue : Le Mois économique et financier (janvier-février 1992). La SBS part d'une analyse macroscopique pour justifier sa position et légitimer le projet : elle constate que la part d'infrastructure de transport dans les grands pays n'a cessé de diminuer depuis le début des années 1970 jusqu'au milieu des années 1980. La construction européenne a renversé la tendance. La SBS salue l'impact démultiplicateur de ces investissements sur le développement économique et explique que 1 '« effet keynésien » de cette politique sur l'emploi place la France dans une meilleure position que la Grande-Bretagne libérale ! Elle se félicite enfin que les États acceptent le recours au financement privé.

Les cantons alémaniques et romands partisans du TGV entrent dans l'association TGV Rhin-Rhône, ainsi que la SBS. En 1995, 9 cantons suisses font partie de la SEM. Il se crée une Association romande pour la promotion du TGV Rhin-Rhône, membre de la SEM. Cela a un effet 
d'entraînement sur les Allemands : le 16 décembre 1992, le Bade-Wurtemberg donne son adhésion à l'association TGV Rhin-Rhône. Le 9 décembre 1992, le ministre des Transports, Jean-Louis Bianco, annonce sa volonté d'engager « les études préliminaires d'un tronçon de ligne nouvelle entre Mulhouse et la Bourgogne ». La SNCF reçoit sa lettre de mission.

Une réunion interministérielle sur les liaisons ferroviaires à grande vitesse a lieu le 4 février 1998, présidée par Lionel Jospin, Premier ministre, dont le compte rendu figure dans le fonds Chevènement. Le ministre de l'Intérieur considère que le TGV Rhin-Rhône est une sorte de « contrepartie » à l'abandon du canal Rhin-Rhône et explique que la « rentabilité » du TGV Rhin-Rhône sera «bien supérieure » à celle du TGV Est. Il craint la « concurrence » d'autres projets de TGV. Dominique Strauss-Khan, ministre de l'Économie, des Finances et de l'Industrie fait observer « qu'aucun projet de TGV n'a de rentabilité économique ». Conciliant, le ministre délégué chargé des Affaires européennes (Pierre Moscovici) demande de faire apparaître que les deux projets de TGV ne s'opposent pas mais se complètent. À la suite de cette réunion, Jospin tranche. Il décide que, sur la base des études d'avant-projet sommaire, les études préparatoires au lancement de l'enquête publique de la première phase (Mulhouse-Dijon) du TGV Rhin-Rhône seront lancées courant 1998. Jean-Pierre Chevènement venait de remporter une victoire décisive contre ceux qui souhaitaient que cette décision n'intervienne qu'après que la première phase du TGV Est soit achevée.

Le 29 octobre 1999 est enfin signée une convention entre le gouvernement français et le Conseil fédéral suisse relative au raccordement de la Suisse au réseau ferré français à grande vitesse. La Confédération helvétique est restée constante dans ses intentions. Son exemplarité est utile pour l'association dans la mesure où elle lui permet de culpabiliser le gouvernement français accusé de lenteur. Ainsi, le 28 septembre 2005, l'association, réunie au Sénat, envoie une adresse au gouvernement en rappelant que d'autres se sont déjà engagés : " La Commission européenne, la Suisse, les régions Alsace, Bourgogne et Franche-Comté se sont d'ores et déjà engagées à participer au financement de la première tranche Est du TGV Rhin-Rhône. Ne manque plus que la réponse de l'État français. Il est aujourd'hui le seul financeur de ce projet à n'avoir toujours pas fait connaitre sa participation ${ }^{25}$. »

La phase préliminaire du projet LGV donne à voir le croisement d'enjeux locaux et globaux ; il révèle le rôle que la Suisse a joué : Jean-Pierre Chevènement souhaite l'impliquer pour légitimer sa démarche ; la Suisse saisit

\footnotetext{
${ }^{25}$ Ce sera fait le 28 février 2006.
}

l'opportunité de la LGV pour sortir de son encerclement. Le syndrome de l'encerclement est ancien et n'est en rien contradictoire avec le complexe de centralité, cher à Kevin Sutton [25]. Ne lit-on pas dans une publication de la Chambre de commerce de Zürich : « Zürich occupe aujourd'hui une excellente position dans les réseaux de transport suisses et internationaux ces atouts n'ont cependant pas été amenés sur un plateau aux Zurichois. Ils sont bien le résultat d'efforts soutenus en vue de la réalisation, au siècle dernier, des principales lignes ferrées $^{26}$ » Il y a indéniablement dans cette déclaration le constat de formes de continuité susceptibles d'éclairer des logiques contemporaines.

\section{France-Suisse : une relation ferroviaire sur le temps long}

Un autre enjeu du Nord-Franche-Comté est oublié dans cette première phase : la question de la ligne Delle-Porrentruy. Question fort ancienne, mais réactivée par l'accession du Jura (canton et République du Jura) à la souveraineté le $1^{\mathrm{er}}$ janvier 1979. Pour le nouveau Canton, la liaison avec la France est vitale : «Plutôt que de voir le problème exclusivement sous un angle international franco-suisse, il faut partir comme pour le Territoire de Belfort de la situation géographique dans un contexte européen interrégional » [26].

D'ailleurs, cette dimension reste d'actualité, à en juger par un article de Transports romands de janvier 2013, qui fait part de la grande inquiétude quant au maintien de la liaison directe TGV-Berne par l'Arc jurassien (Neuchâtel, Pontarlier, Mouchard), au profit d'une liaison via Bâle. La mobilisation concerne nombre de structures consulaires, cantonales, associatives de chaque côté de la frontière, comme si les anciens tropismes ressurgissaient [27].

\section{Une conscience commune de l'enclavement dès le XIX ${ }^{\mathrm{e}}$ siècle}

Dans la région, la «bataille » du rail ${ }^{27}$ commence historiquement dès les premières enquêtes d'utilité publique suite aux ordonnances royales du 10 février 1834 et du 15 février 1835. S'ouvre alors pour le territoire la double perspective de contribuer à relier la Méditerranée au Rhin via une ligne Dijon-Mulhouse et de bénéficier d'une

\footnotetext{
${ }^{26}$ Voir [23].

${ }^{27}$ Une vive tension apparaît entre les villes de Montbéliard et de Belfort, toutes deux avançant des intérêts divergents quant au tracé de la ligne. Les débats parlementaires sont relayés par la presse locale atteignant un point de paroxysme en 1846. Deux tracés entrent frontalement en concurrence, l'un par la vallée du Doubs évitant Belfort, l'autre par la vallée de l'Ognon.
} 
liaison Le Havre-Paris-Belfort. Le rôle des municipalités, comme celui des conseils généraux, s'avère alors essentiel pour peser dans les arbitrages entre tracés concurrents. Localement, ils donnent lieu à une impitoyable rivalité, entre Belfort et Montbéliard. Ces concessions auxquelles s'intéressent les grandes compagnies ferroviaires posent rapidement la question connexe de la liaison avec la Suisse. En effet, une autre enquête d'utilité publique pour la ligne Strasbourg-Bâle suscite de vives inquiétudes au sein de la municipalité belfortaine. Celle-ci craint de voir une partie du trafic à destination de Bâle transiter par Mulhouse qui s'imposerait alors comme véritable carrefour commercial dans le Rhin-sud. En situation de territoire de marge ${ }^{28}$, l'opportunité du chemin de fer et au-delà le repositionnement sur des axes de trafic international s'imposent comme une évidence pour les villes de moyenne importance. Il y va bien sûr de leur survie et de leurs capacités à faire valoir des intérêts communs avec d'autres territoires en situation analogue.

Ainsi, dès le milieu du XIX ${ }^{\mathrm{e}}$ siècle, les forces vives de la Porte de Bourgogne comme celles de la Suisse occidentale font front commun pour favoriser la porosité d'une frontière politique, unissant leurs efforts pour dynamiser notamment les infrastructures de transports.

Ainsi trois années avant l'inauguration en 1858 des lignes Paris-Mulhouse via Belfort et Dijon-Mulhouse, préfigurant l'axe de la première liaison Rhin-Rhône, l'intérêt d'une liaison en direction de Delle et au-delà de Porrentruy en Suisse commence à se cristalliser. Dès lors cette ligne ne va cesser de mobiliser les instances de tous ordres de chaque côté de la frontière. Une longue histoire commune débute avec comme clef de voûte des préoccupations, la question ferroviaire. Même si la modification du projet [29, 30] reste à l'initiative des grandes familles industrielles franc-comtoises (Japy-Frères et $\mathrm{C}^{\mathrm{ie}}$, Viellard-Migeon et $\mathrm{C}^{\mathrm{ie}}$, Boigeol et $\mathrm{C}^{\mathrm{ie}}$ ), les autorités politiques prennent rapidement le relais. C'est une trajectoire inverse à la LGV.

Un premier contact est pris en 1866 pour envisager un véritable désenclavement du pays de l'Ajoie autour de Porrentruy et Delémont et du futur canton du Jura. $A$ contrario, pour toute la région du Nord-Franche-Comté, cette ouverture est susceptible d'offrir de plus lointaines perspectives en direction des Alpes suisses et au-delà de la Méditerranée via les plaines italiennes. L'ouverture du tronçon Montbpliard-Delle par Beaucourt et Morvillars a lieu en 1866 après une dizaine d'années de travaux revenant à la Compagnie Paris-Lyon-Méditerranée dirigée par les Rothschild. Quant au tronçon Belfort-Delle, il est ouvert en 1877, la concession étant revenue à la Compagnie de l'Est

\footnotetext{
${ }^{28} \mathrm{Au}$ cours de l'histoire « l'aménagement du territoire est aussi devenu l'aménagement des territoires ». Voir [28].
}

animée par les frères Pereire. Belfort est en passe de devenir un nœud ferroviaire.

En effet, avec le succès de ces lignes, se profilent d'autres enjeux qui mêlent à la fois des intérêts locaux et des enjeux plus globaux. Le Nord de la Franche-Comté auquel appartient désormais l'ancien arrondissement du Haut-Rhin de langue française draine le trafic Calais-Paris-BelfortDelémont, et s'ouvre en direction de Berne et de Zürich. La séparation des anciennes provinces d'Alsace et de Lorraine, actée par le Traité de Francfort, donne alors à la situation géographique belfortaine une importance autant militaire que commerciale. Jusqu'en 1918, le passage par Delle devient un point de transit d'importance internationale.

\section{La quête d'une transfrontiérité effective}

À la fin du XIX ${ }^{\mathrm{e}}$ siècle, l'orientation des grands courants d'échanges européens par le rail donne lieu à de très longs et redoutables bras de fer qui s'inscrivent au cœur de stratégies géopolitiques d'envergure, pesant parallèlement sur l'avenir économique des territoires traversés. C'est le cas avec la question du percement du tunnel du Lötschberg dont les travaux débutent en octobre 1806.

Le canton de Berne, alors en dehors des principaux axes ferroviaires helvétiques via les tunnels du Gothard (1882) et du Simplon (1905), envisage une ligne qui puisse le placer sur un nouvel axe majeur. Cette volonté de désenclavement ambitionne de relier à la fois l'Allemagne et le Nord-Ouest de la Suisse (dont le Jura francophone) à l'Italie par le Valais puis le tunnel du Simplon. Le percement de l'Oberland bernois se pose une nouvelle fois ${ }^{29}$ en concurrent sérieux $\mathrm{du}$ Gothard mais il risque également de nuire à la région de Lausanne et de Genève « qui redoute très vivement son exécution » [31] en détournant une partie du trafic vers la France par le Nord Jura. Les rivalités s'exacerbent. Entre Genève et Bâle, entre les tenants du Gothard contre ceux du Lötschberg, entre les parties francophone et alémanique : « C'est pour tendre vers ce but que la direction bâloise des chemins de fer fédéraux mieux inspirée, n'aurait pas dû continuer dans le Jura-Nord, les pratiques de l'ancien Jura-Simplon en les aggravant encore par des tentatives saugrenues d'oppression pangermaniste dont le bon sens et le patriotisme de tous les vrais Confédérés suisses ont fait justice »[32] $]^{30}$

Mais ce projet résonne bien au-delà de la Confédération. C'est également la mobilisation générale du côté français

\footnotetext{
${ }^{29}$ Cette question fait écho aux débats sur le tracé d'une éventuelle percée ferroviaire en Suisse dans les années 1850 et 1860.

${ }^{30}$ Comment réconcilier le Gothard et le Loetschberg sans oublier l'Aarlberg, lettre ouverte à M. le Président de la Chambre de commerce de Bâle, avril 1908.
} 


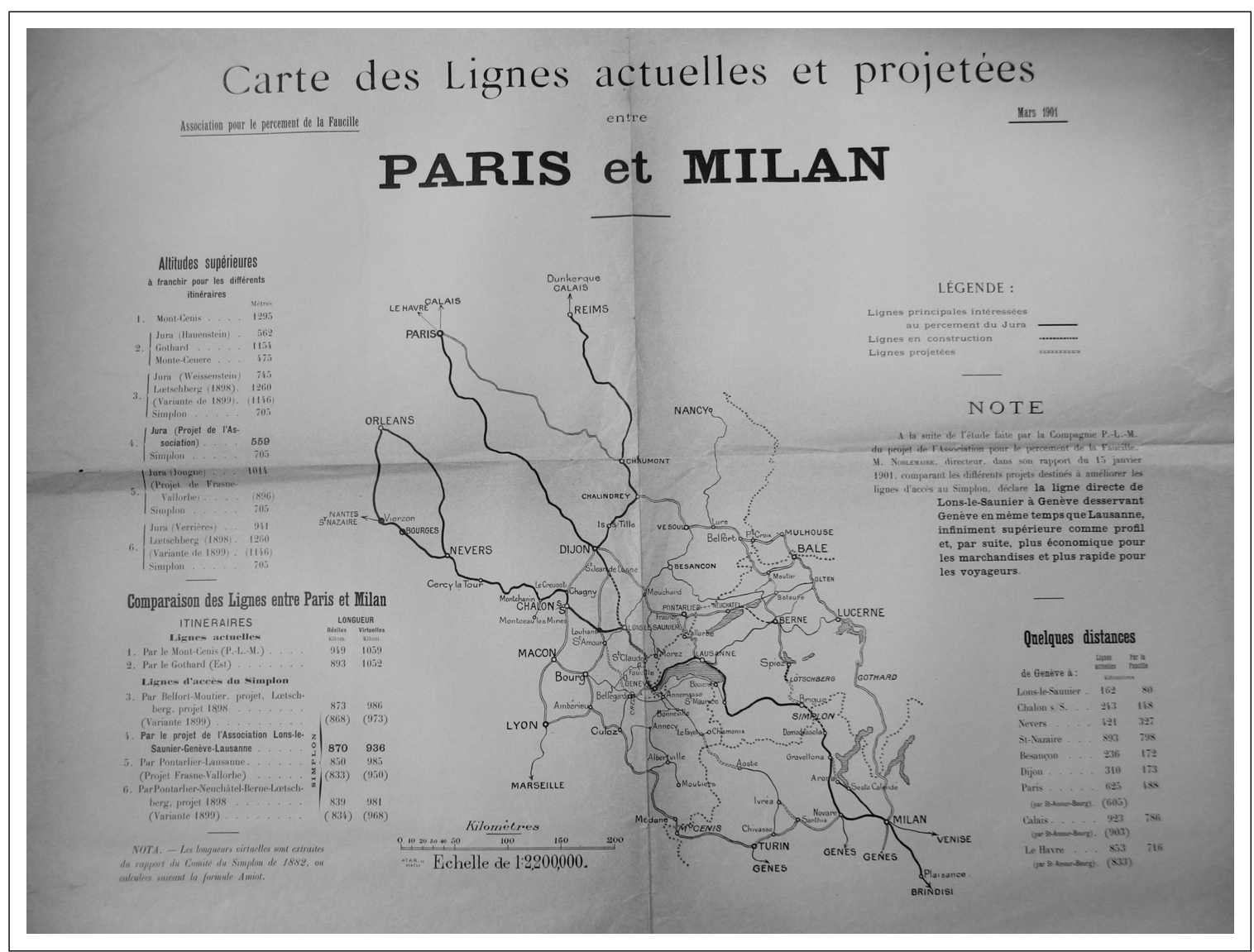

Photographie 1 Projet envisagé par la $C^{\text {ie }}$ PLM favorable à une ligne directe Lons le Saunier-Genève par le percement de la Faucille, mars 1901

ADTB (Archives départementales du Territoire de Belfort), 2 ETP 516.

(Photographie 1)! En effet, les grandes compagnies ferroviaires dans leur concurrence acharnée cherchent à inscrire leur réseau sur de grands axes transeuropéens : Paris-Milan pour la $\mathrm{C}^{\text {ie }}$ PLM (via Frasne et Vallorbe pour desservir Lausanne et Genève), Calais-Milan pour la $\mathrm{C}^{\text {ie }}$ des Chemins de fer de l'Est (via Belfort et Delle en direction de Berne). «Après l'annexion le Jura Bernois s'est trouvé dans une position très avantageuse, favorisée par la convention avec la Compagnie de l'Est concernant l'institution d'un service international d'exploitation. Relié à la Compagnie de l'Est, le Jura bernois sera le chemin d'une grande partie du trafic avec le Nord-Est de la France et de la Belgique » [33]. Dans le même temps, toutes les chambres de commerce françaises (Laval, Moulins, Gray) $^{31}$ susceptibles d'être concernées par ces deux courants d'échanges rivaux sont invitées à se prononcer. Elles rivalisent d'arguments, cartes et chiffres

\footnotetext{
${ }^{31}$ Présence de nombreux facsimilés. La proposition de la Chambre de commerce de Gray, qui défend un tracé par sa ville titre : La question $\mathrm{du}$ Simplon et la ligne directe d'Angleterre et de Belgique en Suisse et en Italie. Voir [32].
}

à l'appui, pour plaider en faveur du meilleur tracé entre Manche/Mer du Nord et Méditerranée. Lettres ouvertes, rapports comparatifs, expertises et contre-expertises [34] se multiplient. La conférence ferroviaire franco-suisse de Berne du 15 mars 1908 concentre l'ensemble de ces aspirations pointant les intérêts convergents des territoires comme autant de révélateurs de synergies potentielles. Les édiles de la ville de Porrentruy « rappellent qu'ils sont les tenants de l'influence française en Suisse et que la ligne du Lötschberg-Simplon où dominent les intérêts français, a été créée pour arracher la Suisse à l'emprise exclusive de l'influence allemande à la suite du percement du Gothard » [35]. De telles déclarations révèlent un sentiment profond qui anime les élites locales.

En France, la Chambre de commerce de Belfort milite parallèlement pour un grand projet, celui du percement des Vosges pour rejoindre plus efficacement Épinal au nom du trafic international vers la Suisse et l'Italie qui se profile : «Il y a lieu de procéder sans retard, tant par les soins de la $C^{\text {ie }}$ de l'Est que par ceux de l'autorité militaire compétente, à l'étude du percement des Vosges au ballon d'Alsace, 


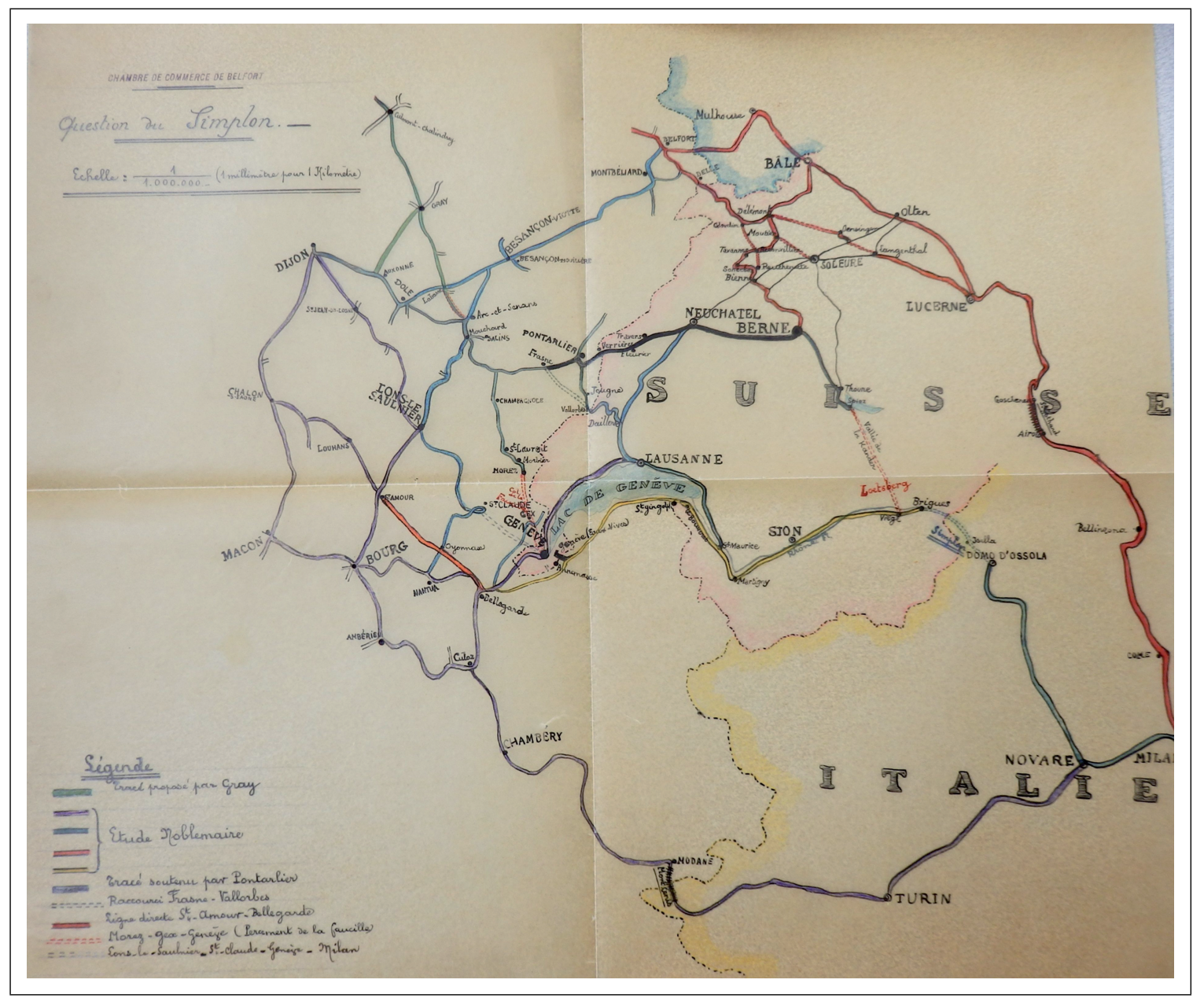

Photographie 2 Document de la Chambre de commerce de Belfort démontrant l'intérêt du tunnel du Loetschberg pour accéder via le tunnel du Simplon à l'Italie au début du $\mathrm{XX}^{\mathrm{e}}$ siècle

ADTB, 2 ETP 516.

pour relier directement Épinal à Remiremont à Belfort et à Delle »[36] ${ }^{32}$. Conscients des enjeux, les décideurs économiques emmenés par Gaston Bornèque, président du Conseil de gérance de Japy-Frères et $\mathrm{C}^{\mathrm{ie}}$ cherchent à consolider les courants d'échanges que les événements de la guerre de 1870 avaient redessinés au profit des activités Belfortaines (Photographie 2). C'est l'opportunité pour le Nord Franche-Comté d'asseoir un nouveau statut dans la géographie nationale. Cette zone de passage aspire à devenir un véritable carrefour d'échanges ! Le développement des infrastructures ferroviaires helvétiques n'est donc pas sans conséquence et reste directement lié aux grandes ouvertures transfrontalières (Photographie 3)!

Le percement des Alpes bernoises qui débute en 1906 débouche sur une exploitation effective de la ligne à partir de

\footnotetext{
32 Auparavant les délibérations du 7 mars 1900, 28 mai 1901, 18 novembre 1902 allaient déjà dans ce sens à savoir un tracé de Calais à Milan au plus court.
}

septembre 1913. L'ouverture vers l'Italie permet d'atteindre une activité record pour la liaison Delle-Porrentruy, portant le nombre de trains quotidiens de 25,5 en 1903 à 41,6 dix années plus tard $[37]^{33}$ ! Le trafic marchandises atteint alors 520000 tonnes. Mais cette montée en puissance est brutalement stoppée par les conséquences de la Première Guerre mondiale avec le retour aux frontières de 1870.

\section{Le retour des intérêts locaux : le rôle de la «question jurassienne »}

La défaite des empires centraux modifie profondément les volumes et les orientations du trafic ferroviaire. Avec le retour de l'Alsace dans le giron français et la création d'une nouvelle compagnie privée (les Chemins de

\footnotetext{
${ }^{33}$ Les intérêts du Jura, bulletin de l'Association pour la défense des intérêts du Jura, XXXI ${ }^{\mathrm{e}}$ année, ${ }^{\circ}$ 5, mai 1960, p. 99.
} 


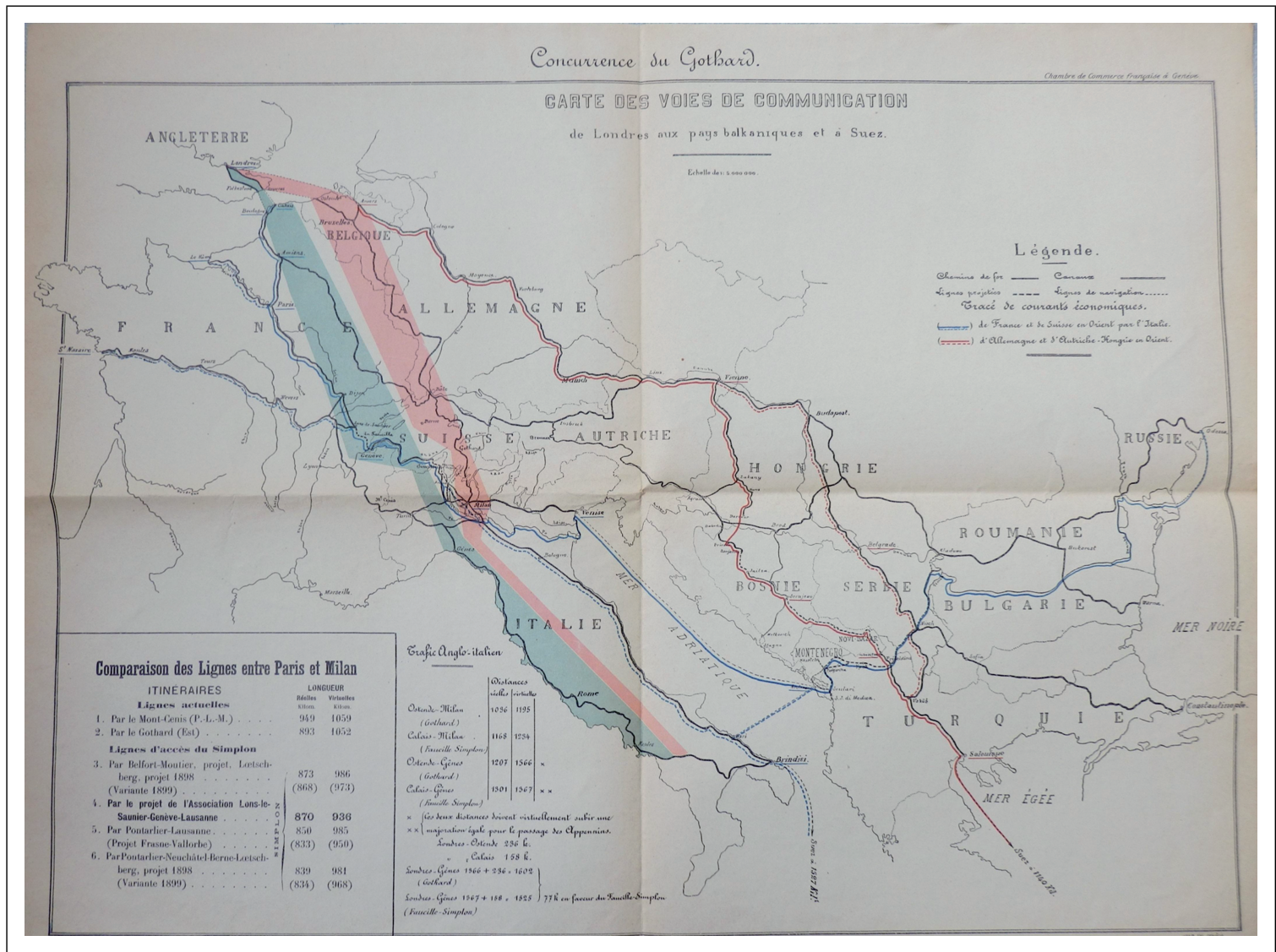

Photographie 3 Tracé des courants économiques d'Allemagne et de France en direction de l'Orient. Document de la Chambre de commerce française à Genève vers 1900 avant le percement du tunnel du Loetschberg

ADTB, 2 ETP 516.

fer d'Alsace-Lorraine), la ligne Strasbourg-Mulhouse-Bâle concurrence à nouveau par son orientation Nord-sud, la liaison avec la région du Nord et la Belgique. De plus la nationalisation des chemins de fer en 1937 (qui fusionne les sept grandes entreprises ferroviaires pour donner naissance à la SNCF) ajoute aux tendances en cours. En effet, une nouvelle politique du rail se met en place tendant à privilégier les lignes les mieux fréquentées. Au nom de la rentabilité, certaines lignes considérées désormais comme secondaires ne sont plus prioritaires. La situation s'aggrave un peu plus au lendemain de la Seconde Guerre mondiale avec la remise en état du réseau national. C'est le cas pour le tronçon Belfort-Delle qui ne bénéficie pas du plan d'électrification, alors que les chemins de fer suisses sont électrifiés jusqu'à la frontière de Delle.

Ce brusque retour à une marginalité de fait va paradoxalement « nourrir » une stratégie volontariste mais de l'autre côté de la frontière. En effet, la baisse de la fréquentation de la ligne reste significative. En 1936, le volume fret tombe à 90000 tonnes. En octobre 1938, c'est la suppression des trains omnibus entre Belfort et Delle. La tendance à la désaffection s'accentue encore avec l'interruption complète du trafic pendant la guerre et avec la destruction de la gare de Delle en 1944. Le fret ne représente plus que 33000 tonnes en 1953. Le nombre de trains quotidiens entre Delle et Belfort se réduit à huit. Cette déshérence pousse les autorités cantonales bernoises à réagir vivement constatant l'abandon de leur seule voie ferroviaire internationale : « Il ressort de cette étude qu'il faut que le Jura revendique, avec la dernière énergie, un intérêt pour la ligne de Delle [...]. Il faut sur le plan fédéral, réclamer que subsiste cette grande transversale ferroviaire qu'est le Milan-Paris passant par Berne » [38].

Alors qu'au niveau de la Confédération la question d'un risque de marginalisation de la Suisse est écartée grâce à l'accord passé avec la France du 11 mai 1954, qui prévoit une participation financière ${ }^{34}$ à la mise en place de la traction électrique sur des lignes transfrontalières, le canton de Berne manifeste son désarroi quant à la transversale Paris-Milan par le Jura. Les échecs successifs

\footnotetext{
${ }^{34}$ Cette participation financière se monte à 250 millions de francs suisses pour les lignes Strasbourg-Bâle, Dijon-Vallorbe, PontarlierLes-Verrières.
} 
de plusieurs interventions au Conseil fédéral et la tenue d'une grande conférence mettent en exergue l'inertie des autorités françaises : " la question de l'aménagement $\mathrm{du}$ tronçon Belfort-Delle ne pouvait se poser tant que la France n'envisageait pas l'électrification de la grande ligne Paris-Belfort-Bále. C'est seulement à ce moment que la Suisse pourra examiner la possibilité de moderniser la ligne de Delle $»^{35}$. Cependant, le canton de Berne obtient l'autorisation quelques mois plus tard de faire procéder par la Direction générale des Chemins de fer fédéraux, à une étude technique concernant l'électrification de la ligne Belfort-Delle avec une estimation des coûts induits ${ }^{36}$.

Cette stratégie de lobbying est relayée par l'ADIJ ${ }^{37}$, puissante association fondée en 1925, ayant pour objectif de faire reconnaître les richesses régionales ainsi que de défendre politiquement le Jura et de lutter contre son enclavement : " Pour nos cités jurassiennes où l'industrie connaît un bel essor [...] la rapidité et la commodité des transports jouent un grand rôle. Ainsi le Jura, Bienne, voire Granges et Soleure, y compris la ligne Moutier-Soleure, subissent un préjudice certain de la situation actuelle ${ }^{38} »$. Les autorités helvétiques déplorent le peu d'appui trouvé jusque-là, surtout du côté de la SNCF « qui semble vouloir contourner la région $»^{39}$ ! Le Jura suisse lie son destin à celui du Nord Franche-Comté : « Il y va de l'intérêt public et de la sauvegarde économique de toute une région » qui s'étend de chaque côté de la frontière. Cette problématique, comme on l'a vu, est réactivée avec la LGV. Le constat est très lucide, justifiant une réelle volonté d'action : «Il n'est donc pas exagéré de prétendre que si la ligne Paris-Belfort n'est pas électrifié, le glas de la ligne de Delle, en tant que ligne de trafic international aura bientôt sonné. Nous ne pouvons admettre que ce soit le sort inévitable de cette ligne. Elle est d'une importance telle pour notre région, pour le canton de Berne, pour la ligne du Simplon aussi, que nous ferons tout ce qu'il est possible d'entreprendre pour lui assurer une destinée moins sombre ${ }^{40} »$. Il est déjà essentiel d'insérer le territoire dans les grands réseaux d'échanges européens et ainsi de valoriser sa position géographique.

\footnotetext{
35 Réponse du chef du Département fédéral des Postes et des Chemins de fer en date 6 mai 1954, adressée à l'Association pour la défense des intérêts du Jura. Voir [37 p. 101].

${ }^{36}$ Rapport du 20 janvier 1956 : la ligne Paris-Delle-Berne-Milan est clairement évoquée.

${ }^{37}$ Association pour le Développement et l'Initiative dans l'Arc Jurassien.

${ }^{38}$ Déclaration de M. CH. Parietti, député et maire de Porrentruy, extrait du procès-verbal du 16 décembre 1956 entre une délégation du gouvernement et une délégation jurassienne. Voir [37].

${ }^{39}$ Propos de M. Moeckli, Conseiller d'État, délégué au gouvernement pour les questions ferroviaires. Voir [41].

${ }^{40}$ Lettre du président de l'ADIJ à la Chambre de commerce de Belfort du 8 juillet 1955 . Voir [42].
}

L'alerte en direction des institutions belfortaines est sans concession, appelant à la revendication et à l'action. La stratégie est même insufflée à la demande de l'instance française ${ }^{41}$ par une élite jurassienne vent debout : « Ce sont tous les groupements économiques intéressés entre Troyes et Belfort qui devraient s'unir pour exiger l'électrification de la ligne Paris-Belfort-Delle » [44]. Au-delà d'une critique à peine voilée d'une stratégie économique timorée, cet épaulement mutuel franco-suisse sera le socle de l'aventure LGV Rhin-Rhône. A contrario des « années » Chevènement, la volonté de créer, d'organiser une force de pression institutionnelle et légitime par-delà les frontières est à l'initiative des édiles suisses.

Chaque épisode est relayé dans le détail par la presse (Tribune jurassienne, Jura libre, Le Démocrate, L'Impartial) qui consacre de longs articles aux débats et aux prises de position respective. À partir 1974, le vote d'autodétermination qui consacre la naissance du canton du Jura francophone par séparation avec le canton de Berne ravive la question de la ligne de Delle. "Le principal handicap de cette région à l'heure actuelle réside précisément dans une insuffisance manifeste des infrastructures de transports $\gg^{42}$. L'impératif de désenclavement passe par la possibilité d'une ouverture performante à la fois routière ${ }^{43}$ et ferroviaire sur la France. Le rapport Boillat (1987) s'inscrit bien dans cette revendication à la fois identitaire et ferroviaire.

Côté français la mobilisation souffre d'une anémie des initiatives. Un Comité d'action franco-suisse de défense de la ligne de Delle mis en place depuis 1947 agit pour la construction d'une gare internationale à Delle. La Chambre de commerce de Belfort appuie la demande [40] en affirmant la nécessité de réduire la durée du trajet Paris-Berne-Lötschberg-Brigue via Delle !

Seule l'instance consulaire se fait véritablement l'écho des aspirations cantonales Jurassiennes, relayées elles par de nombreuses autorités politiques (Communes du district de Porrentruy, Grand Conseil bernois, Conseil fédéral) et portées par plusieurs institutions suisses (Union cantonale de l'industriel et du commerce de Bienne-Jura, Union $\mathrm{du}$ commerce et d'industrie de l'Ajoie et du district de Delémont, Comité des treize). Finalement seules les municipalités proches de la frontière comme Delle ou Grandvillars prennent des positions plus radicales au moins dans les discours. À plusieurs reprises devant ces

\footnotetext{
41 « Lors de notre dernière entrevue vous nous avez priés de vous suggérer un plan d'action. Nous ne saurions en formuler un autre. L'enjeu est important, les moyens à mettre en œuvre doivent l'être aussi ». Lettre du Président de l'ADIJ adressée à M. le Président de la Chambre de commerce de Belfort, 8 juillet 1955. Voir [32].

42 Cabinet du Conseil général, rapport service des transports et de l'énergie, République et Canton du Jura, mars 1984. Voir [39].

${ }^{43}$ Il s'agit du projet de la Transjurane aujourd'hui en cours d'achèvement.
} 
déclarations d'intention, la SNCF donne l'assurance que le point de transit dellois sera maintenu. En réalité, le statu quo demeure laissant la situation de la ligne lentement se dégrader faute d'une desserte convenable et d'un tonnage annuel de marchandises stagnant aux environs de 300000 tonnes. L'année 1975 voit rouler les dernières voitures directes Berne-Bienne-Paris. La suppression des trains express intervient en 1992 au nom de la rentabilité. Une année plus tard, les trains de marchandises se limitent désormais au tronçon Belfort-Grandvillars et en 1995, malgré de nouvelles études d'exploitation et de coût-utilité d'une liaison électrifié de Delémont à Belfort, c'est la fermeture de la gare de Delle.

\section{Réactivation de la ligne Delle-Porrentruy à la faveur de la LGV}

Cette décision intervient alors que d'autres batailles de longue haleine s'engagent ou se durcissent. Côté suisse, la stratégie d'effacement des goulets d'étranglement entre les frontières italiennes et franco-allemandes provoque « La seconde bataille du Lötschberg » $[41]^{44}$. Des représentants français sont à nouveau présents dans des associations de lobbying comme le Conseil des Trente ${ }^{45}$ qui entend peser dans les études de la Commission Transalp 2005 sur la question du choix des grands tunnels de base sous les Alpes.

En France, la demande de l'association Trans-EuropeTGV vise à prolonger les effets de la grande vitesse sur Milan, via le Simplon, sur fond de volonté de désenclavement de la Franche-Comté et de l'Arc Jurassien. « En d'autres termes, à défaut d'initiatives communes menées à bien, ces régions placées à l'écart de tout investissement TGV seront incapables, à terme, de soutenir la comparaison économique avec les autres régions européennes aujourd'hui d'importance semblable, déjà un peu desservies de façon moderne ${ }^{46} \gg$. Les effets de la grande vitesse constatés sur les territoires sillonnés depuis deux décennies, ont été clairement appréhendés par les collectivités territoriales et cette prise de conscience se traduit par des stratégies beaucoup plus volontaristes, ce qui ne peut que réjouir les instances du canton du Jura!

La question de la réouverture de la ligne de Delle revient « naturellement » au cœur de préoccupations interrégionales

\footnotetext{
${ }^{44}$ Devant le succès de la ligne dont les capacités sont aussi aujourd'hui saturées, il s'agit d'achever le doublement intégral de la voie sous le tunnel qui demeure à voie unique sur les $2 / 3$ de la longueur. Certes il faut préciser que ce n'est pas non plus la préoccupation principale suisse en termes de tunnels. L'objectif est d'achever l'équipement du Gothard de base dans les temps.

${ }^{45}$ Sont présents Jean Monnier, vice-président du Conseil général du territoire de Belfort en charge des transports, M. Lehmann, conseiller général et délégué de la CTJ.

${ }^{46}$ Rapport du 31 mai 1988. Voir [42].
}

transfrontalières. Cette fois-ci la mobilisation est effective et symétrique de chaque côté de la frontière. C'est désormais dans le cadre de la Communauté de travail du Jura (CTJ) constituée en $1985^{47}$ que les élus se rencontrent régulièrement. Cette institution se donne pour objectif de développer les synergies dans l'Arc jurassien avec pour principale préoccupation les infrastructures de transport sur fond de «préparation au grand remodelage économique européen ${ }^{48}$. Il s'agit déjà de créer un espace revendicatif au moment où Jean-Pierre Chevènement occupe le terrain politique avec le lancement de LGV Rhin-Rhône. En octobre 2001, l'Association de la ligne ferroviaire Delémont-Belfort est créée. Présidée à sa naissance par Raymond Forni ${ }^{49}$, elle entend obtenir la réouverture de la ligne en $2010^{50}$. L'argumentaire au-delà de la mise en correspondance avec la future LGV Rhin-Rhône pour les voyageurs reprend la perspective de la grande transversale Paris-Milan pour le fret. Des études sont programmées et des crédits octroyés avec un premier acte symbolique qui passe par la réouverture de la gare de Delle le 10 décembre 2006. Cet événement permet aux trains Regio-Express en provenance de Bienne de passer Boncourt et de parcourir 1200 mètres supplémentaires avec un terminus en France ! En 2010 le financement de la revitalisation de la ligne Delle-Belfort est bouclé à hauteur de 100 millions d'euros avec un accès aux quais de la voie LGV et une électrification $\mathrm{du}$ tronçon, plus d'un demi-siècle après les premières « injonctions » helvétiques ! Belfort ne sera plus qu'à cinq heures de Milan.

\section{Conclusion}

L'histoire nous révèle que la question du TGV Rhin-Rhône s'inscrit dans une relation partenariale séculaire avec la Suisse et plus particulièrement avec sa partie Nord-Ouest, jurassienne et francophone. Le « paradigme » helvétique a constamment été présent dans la redéfinition des espaces territoriaux et des enjeux liés à l'évolution des infrastructures de transport. On observe une continuité frappante en

\footnotetext{
${ }^{47}$ La région de Franche-Comté d'une part, les quatre cantons du Jura, de Berne, de Vaud et de Neuchâtel d'autre part signèrent le 3 mai 1985, à Delémont, la Convention instituant la Communauté de travail du Jura. ${ }^{48}$ Compte rendu de la rencontre des élus du Comité de la Communauté de travail du Jura, 26 août 1988. Voir [42].

${ }^{49}$ Maire de Delle (1995-2004), conseiller général du canton de Delle (1987-2001), Raymond Forni obtient un second mandat de député en 1997. Après deux vice-présidences à l'Assemblée nationale, il la présidera de 2000 à 2002, étant alors le quatrième personnage de l'État. 50 « Forte des appuis du Territoire de Belfort et des cantons de Berne, Bâle-Ville et du Jura, d'une quarantaine de communes suisses et françaises et des milieux de l'économie, la jeune association de droit français a enregistré samedi deux soutiens de taille, avec les adhésions annoncées des villes de Bienne et Moutier. » Le Journal du Jura, $1^{\text {er }}$ octobre 2011.
} 
matière de mobilité et de transports, dans cette course à la contraction des espaces. Ainsi, dès le milieu du XIX ${ }^{\mathrm{e}}$ siècle, le paradigme helvétique s'insère au cœur des politiques de développement du ferroviaire dans cette marche de l'Est que constitue le Nord Franche-Comté. Une communauté de destin entre des espaces enclavés se consolide au travers des dossiers d'infrastructures majeures, tant du côté suisse avec le percement du Lötschberg, que du côté français avec la liaison Rhin-Rhône.

Il faut rappeler que «pour être à grande vitesse, les réseaux ferroviaires doivent d'abord être des réseaux à grande distance » [43]. En effet, cette relation d'épaulement franco-suisse procède d'une logique à double échelle, à savoir la nécessité d'une mise en résonance et en réseau des territoires locaux, effaçant la frontière, cherchant dans le même temps à répondre à des enjeux forts liés à de grandes transversales européennes (Nord-Sud et Est-Ouest). Cet emboîtement des intérêts régionaux et transnationaux qui demeurent indissociables, s'affirme au cours du temps comme une constance. Un héritage des similitudes se construit à l'analyse des sollicitations transfrontalières réciproques dans les stratégies mises en place comme dans les postures adoptées au cours des processus décisionnels. Le tropisme allemand reste un facteur cohésif déterminant pour engager des actions institutionnelles d'envergure, même si au bout du compte l'adhésion de cantons alémaniques pour un raccordement au réseau grande vitesse européen s'avère décisive.

$\mathrm{Si}$, dans l'instruction et l'avancée des dossiers, la référence aux grandes diagonales transnationales constitue le cœur de la stratégie argumentative, elle ne doit pas cacher des batailles plus locales mais tout aussi vitales. Deux échelles sont à conjoindre. La réalité de ces combats déterminants ne doit pas être occultée. Ainsi, l'histoire de la ligne Belfort-Delle-Porrentruy reste révélatrice de fortes solidarités entre deux bassins territoriaux. Elle symbolise la vision complémentaire de deux hommes politiques au caractère bien trempé : Jean-Pierre Chevènement et Raymond Forni, l'un privilégiant la dimension européenne, le second se cantonnant dans une approche davantage "localiste » animée d'un certain pragmatisme territorial. Mais au-delà de cette différence de positionnement, l'interconnexion avec la grande vitesse permet aux territoires de marge d'aspirer à devenir des carrefours d'échanges. Ces espaces périphériques (Nord-Franche-Comté, canton du Jura), représentant ici environ 400000 habitants, se sont saisis de l'opportunité d'un grand réseau de transports comme une des conditions d'affirmation de leur présence au cour de l'Europe.

Enfin, les grands projets ferroviaires exigent patience, ténacité et intelligence stratégique. L'infrastructure elle-même n'est qu'une composante d'un projet global.
Jean-Pierre Chevènement l'a bien compris dans la construction de la viabilité dynamique de la liaison Rhin-Rhône. En « empruntant » à l'histoire, en faisant émerger une vision partagée et en entretenant des relations partenariales fortes via une gouvernance transfrontalière du montage financier, il a contribué à consolider un lien fédérateur au sein de l'Arc jurassien franco-suisse. Reste à savoir si ce lien pourra faciliter l'émergence de nouveaux espaces.

\section{Références}

1. Archives Municipales de Belfort (AMB), fonds Jean-Pierre Chevènement, $18 \mathrm{Z} 163$.

2. Offner JM (1993) Les effets structurants du transport : mythe politique, mystification scientifique. L'Espace géographique 3 : 233-242.

3. Lamard P, Stoskopf N (2011) Transports, territoires et société, Éditions Picard, $275 \mathrm{p}$.

4. Plassard-Buguet $F$ (mars 1989) Infrastructure de transport et transformations de l'espace. Le cas de la région du Creusot et de Montceau-les-Mines entre 1870 et 1980. Culture technique 19s : 158.

5. Plassard F (2003) Transport et territoire, La Documentation française.

6. Belot R (2007) Humaniser et universitariser la technologie. L'enjeu de la création des universités de technologie. In : Faucheux M, Forest $\mathrm{J}$ (dir.) Les recherches en sciences humaines et sociales dans les écoles d'ingénieurs éd. Petra, Paris, pp. 75-89.

7. Lamard P, Lequin YC (2006) La technologie entre à l'université : Compiègne, Sevenans, Belfort-Montbéliard, coll. « Sciences humaines et technologie », Belfort, UTBM, $392 \mathrm{p}$.

8. Sétra (2009) Transport ferroviaire régional à grande vitesse. Des exemples européens, Rapport d'études, juin.

9. Mignot J-L (2011), L'arrivée de la grande vitesse ferroviaire dans l'Est de la France : un espoir de désenclavement de la Haute-Saône par un renouveau des axes méridiens ?, thèse de doctorat en géographie, sous la direction d'André Humbert, 23 novembre, Nancy.

10. Rissoan JP (1995) Chronique rhodanienne : le canal Rhin-Rhône, débat sur un projet. Revue de géographie de Lyon 70(1) : 77-79.

11. Belot R (dir.), Hauser C, Python F, Tissot L (2006) Guerres et frontières. La frontière franco-suisse pendant la Seconde Guerre mondiale, Actes du colloque international Histoire(s) de frontières. L'espace jurassien à l'épreuve de la Seconde Guerre mondiale (1939-1945), 22-23 avril 2005, Porrentruy (Suisse), universités de Belfort-Montbéliard, de Fribourg et de Neuchâtel, éd. Lavauzelle, Paris-Panazol.

12. Mémoire, territoire et histoire. Retour sur le colloque de Lucelle en guise de conclusions scientifiques. In : Des deux côtés de la frontière : le Jura bernois, les régions françaises et alsaciennes avoisinantes (1939-1945), Actes du colloque franco-suisse du 29 avril 2006 à Lucelle, Porrentruy (Suisse).

13. Lamard P (2007) Le pays de la grande industrie. In : Daumas JC, Tissot T (dir.), L'Arc jurassien, histoire d'un espace transfrontalier, Maé-erti Éditeurs, Vesoul, pp. 174-178 et pp. 264-267.

14. Lamard P, Woessner R (2007) Rhin Sud, un territoire en devenir ? Pôle Éditorial UTBM, coll. « Territoriales », Montbéliard, 274 p.

15. Benz G (1998) La route et le rail en Suisse, 150 ans de contradictions entre les intérêts régionaux, sectoriels, idéologiques et le défi européen. Relations internationales $96: 451-469$.

16. AMB, rapport du groupe de travail franco-allemand (1987). 
17. Menerault P (1997) Le TGV-Nord est-il soluble dans les figures de la concertation à la française ? Les Cahiers scientifiques $d u$ Transport $32: 96$.

18. Ollivier-Trigalo M (2000) Les grands projets de transport transeuropéens : multiplicité des acteurs, conflits et coordination de l'action. Les Cahiers scientifiques du Transport $37: 24$.

19. Chevènement JP (2011) La France est-elle finie ? Fayard, p. 111, p. 211.

20. Brunet R (janvier-mars 1993) L'Arc atlantique, son reflet et ses mirages. Norois 157 : 19-34.

21. Woessner R. (2004) Le Grand-Est français, une région incomplète. Les Cahiers scientifiques du Transport 46: 45-62.

22. Lettre (à en-tête de l'association Trans Europe Rhin-Rhône Méditerranée) de Jean-Pierre Chevènement à Mathias Feldges, Belfort, 14 juin 1991.

23. Zürich Pro TGV. Comment aller plus loin ?, Plaquette réalisée à l'occasion de la célébration, le 11 octobre 1991 à Zürich, du $75^{\mathrm{e}}$ anniversaire de la fondation de la société Züblin AG, avant-propos.

24. Note de Thierry Zettel à Jean-Pierre Chevènement, président de Trans-Europe TGV, Belfort, 24 octobre 1991.

25. Sutton K (2011), Les nouvelles traversées alpines : entre co-spatialité de systèmes nationaux et recherche d'interspatialité, une géopolitique circulatoire, thèse en géographie, Université de Grenoble, décembre.

26. ADTB, La ligne Bienne-Delémont-Delle-Belfort dans une perspective européenne interrégionale transfrontalière, République et canton du Jura, service des transports et de l'énergie, fonds Jean Monnier non classé.

27. TGV Berne-Paris : grande mobilisation, Transports romands 16, janvier 2013, p. 15.
28. RITMA, Regards croisés sur les territoires de marge(s), Presses universitaires de Strasbourg, 2001, p. 7.

29. ADTB, Projet du Chemin de fer de Montbéliard à Delle par Audincourt et Beaucourt, 58 S1 et 5S 206.

30. Gavoille J (1977), Transports et voies de communications, colloque Association interuniversitaire de l'Est, Dijon, mars.

31. Zimmermann M (1903) Les voies d'accès au Simplon. Premières voies suisses et italiennes. Annales de Géographie 12(64) : 374.

32. ADTB, 2 ETP 516.

33. Les intérêts du Jura 5, bulletin de l'Association pour la défense des intérêts du Jura, XXXIe année, mai 1960, p. 98.

34. Zimmermann M (1905), Les voies d'accès au Simplon et le tunnel de la Faucille. Annales de Géographie 14(73) : 87-90.

35. AMB, 20 207, note relatant la visite de la municipalité de Delle à Porrentruy en date du 23 janvier 1948.

36. Extrait du registre des délibérations de la Chambre de commerce de Belfort du 20 juin 1908.

37. ADTB 2 ETP 525.

38. La Tribune jurassienne 278, D'importants problèmes intéressant le Jura discutés à l'assemblée du comité de l'ADIJ, à Granges, 26 novembre 1953.

39. ADTB, $1578 \mathrm{~W} 27$.

40. Debrot J (1960) Rapport mars, Chambre de commerce de Belfort.

41. Transports romands 9, Bulletin d'information sur les transports publics de suisse romande et de France voisine, avril 2011.

42. ADTB, $1545 \mathrm{~W} 72$

43. Ollivro J (1995) Le réseau des lignes à grande vitesse : prégnance centralisatrice ou redéfinition de l'espace français ? Revue d'histoire des chemins de fer 12-13 : 204 p. Printemps automne. 